\title{
Newly Located Occurrences of Lode Gold Near Table Mountain, Circle Quadrangle, Alaska
}

U.S. GEOLOGICAL SURVEY BULLETIN 1682

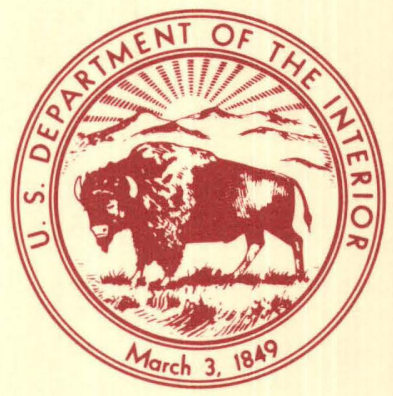





\section{Newly Located Occurrences of Lode Gold Near Table Mountain, Circle Quadrangle, Alaska}

By W.D. MENZIE, RENMIN HUA, and H.L. FOSTER

Results of brief studies of the field relations, trace-element geochemistry, mineralogy, and fluid inclusions of new lode gold occurrences near Table Mountain, Circle quadrangle, Alaska, are reported. Similar occurrences probably were the sources of placer gold deposits in the Circle mining district. 


\title{
DEPARTMENT OF THE INTERIOR \\ DONALD PAUL HODEL, Secretary
}

\author{
U.S. GEOLOGICAL SURVEY
}

Dallas L. Peck, Director

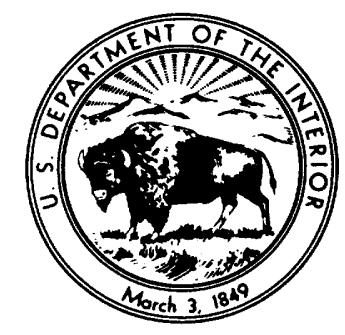

For sale by the

Books and Open-File Reports Section

U.S. Geological Survey

Federal Center, Box 25425

Denver, CO 80225

Library of Congress Cataloging-in-Publication Data

Menzie, W.D.

Newly located occurrences of lode gold near Table Mountain, Circle quadrangle, Alaska.

(U.S. Geological Survey Bulletin 1682)

Bibliography: $\mathrm{p}$.

Supt. of Docs. No.: I 19.3:1682

1. Gold ores-Alaska Mountain Region (Yukon-Koyukuk Borough). I. Hua, Renmin. II. Foster, Helen Laura, 19191682 .

QE75.B9 No. 1682

[QE390.2.G65]

$557.3 \mathrm{~s}$ [553.4'1'097986]

86-600235 


\title{
CONTENTS
}

\author{
Abstract $\mathbf{1}$ \\ Introduction $\mathbf{1}$ \\ Geologic setting 2 \\ Results of initial geochemical sampling 4 \\ Field relations of the gold occurrences 4 \\ Geochemical results 4 \\ Petrography of mineralized samples 4 \\ Fluid-inclusion studies $\mathbf{5}$ \\ Crushing experiments $\mathbf{9}$ \\ Discussion 10 \\ References cited $\mathbf{1 3}$
}

\section{FIGURES}

1. Map of the Circle quadrangle 1

2. Reconnaissance geologic map of the Table Mountain area 2

3. Photomicrographs of selected thin and polished sections from the Table Mountain a rea 3

4. Map showing locations of sample sites $\mathbf{5}$

5. Histograms of homogenization temperatures of fluid inclusions from the Table Mountain occurrence 12

6. Histograms of homogenization temperatures of fluid inclusions from occurrences in and adjacent to the granite $\mathbf{1 2}$

\section{TABLES}

1. Analytical data of rocks from the Table Mountain area 6

2. Analytical data of rocks from stations 31,32 , and 33 in the Table Mountain area collected in 1983 


\title{
Newly Located Occurrences of Lode Gold Near
}

\section{Table Mountain, Circle Quadrangle, Alaska}

\author{
By W.D. Menzie, Renmin $\mathrm{Hua}^{1}$, and H.L. Foster
}

\begin{abstract}
Newly located occurrences of lode gold in the vicinity of Table Mountain, Circle quadrangle, are probably similar to those which were sources for placer gold in the Circle district and Faith Creek area. Brief studies were made of the field relations, trace-element geochemistry, mineralogy, and fluid inclusions of the occurrences. Most of the goldbearing samples are clustered in two areas. The highest concentrations of gold $(2.6-140 \mathrm{ppm})$ occur, just west of Table Mountain, in black biotite schist and in quartz veins adjacent to a fault zone that is intruded by a hypabyssal felsic dike. Five kilometers to the northeast, where a small granite pluton crops out, gold was detected in lesser amounts ( 0.05-0.2 $\mathrm{ppm}$ ) in country rocks adjacent to the granite, in the granite adjacent to dikes, and in felsic dikes. The occurrences have a simple mineralogy: pyrrhotite, arsenopyrite, minor chalcopyrite, and rare enargite and sphalerite. They formed from low-saline fluids ( 10 weight percent $\mathrm{NaCl}$ equivalent) whose sulfur fugacities were within the stability field of pyrrhotite. Based upon fluid-inclusion data, we infer that the occurrences formed at moderate temperatures from fluids that initially boiled, over the range 370 to $320^{\circ} \mathrm{C}$, and then gradually cooled. Field relations and estimated depths of formation of cont act metamorphic effects and quart $z$ veins indicate that the occurrences formed in an active tectonic environment.
\end{abstract}

\section{INTRODUCTION}

The Circle quadrangle, a major gold producing area of Alaska, has produced at least $850,000 \mathrm{oz}$ of gold (Bundtzen and others, 1984), all from placer deposits. Production has been from four areas (Menzie and others, 1983), the majority coming from the Circle mining district. During regional geologic studies, which were part of the Alaska Mineral Resource Assessment Program (AMRAP), several samples of

\footnotetext{
${ }^{1}$ Department of Geology, Nanjing University, Nanjing, China.
}

gold-bearing rock were collected from two areas in the Circle B-4 quadrangle. At one area, near Table Mountain, two samples of fine-grained biotite schist collected adjacent to a fault contained 40 and $140 \mathrm{ppm}$ gold. About $5 \mathrm{~km}$ northwest of Table Mountain, low levels of gold $(<1 \mathrm{ppm})$ were detected from samples taken in and adjacent to a small felsic pluton.

Although the streams that drain these two areas have not been mined, the main productive streams of the Circle district are just to the east; a smaller area of placer mining, here called the Faith Creek placer area, lies to the west of Table Mountain (fig. 1). Because occurrences such as those near Table Mountain and the pluton are probably similar to those that were sources for the placer gold, brief studies were made of the field relations, trace-element chemistry, mineralogy, and fluid inclusions. The studies indicate that these gold occurrences, which are characterized by a simple mineral assemblage, probably formed at shallow depths in and adjacent to fractures that were active during the late stages of felsic igneous activity.

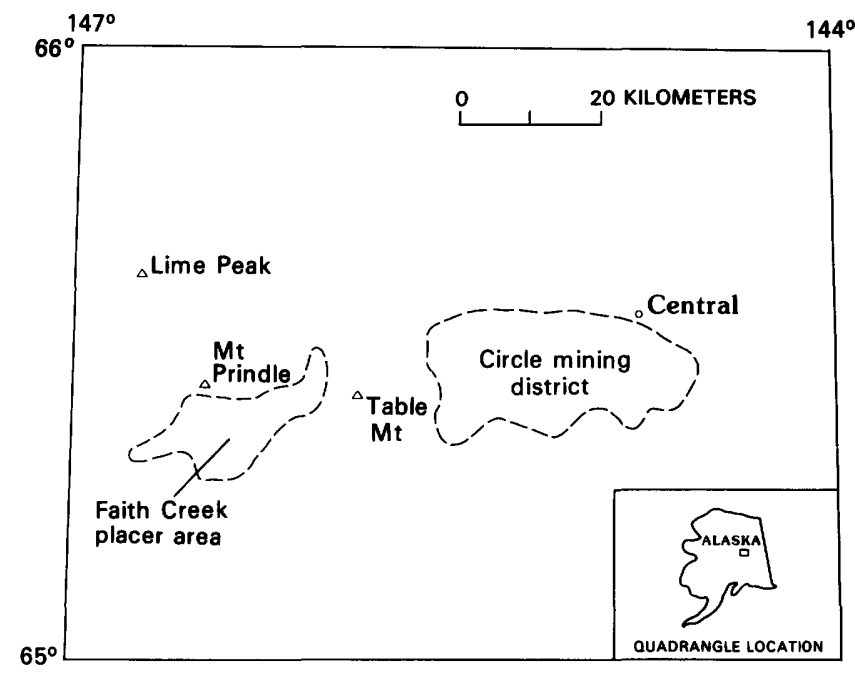

Figure 1. Circle quadrangle showing locations of areas discussed in text. 


\section{GEOLOGIC SETTING}

The Table Mountain area, Circle quadrangle, is in the northeastern part of the Yukon Tanana Upland-- a physiographic province that mostly lies between the Yukon and Tanana Rivers in east-central Alaska. Recent geologic studies include reports by Foster and others (1983), Burack and others (1984), and Burack (1983). The Table Mountain area (fig. 2) is composed primarily of pelitic and quartzitic schists and quartzites that have been regionally metamorphosed to upper greenschist facies, then subsequently intruded and contact metamorphosed by a granite (Streckeisen, 1975) pluton. The rocks were in turn cut by a leftlateral fault (the Swamp Saddle fault) and intruded by felsic hypabyssal rocks, probably in the early Tertiary.

The metamorphic rocks in the Table Mountain area belong to the quartzite and quartzitic schist unit of Foster and others (1983) and are composed of light gray quartzite, black biotite schist, fine-grained greenish-gray mafic rocks and light-greenish-gray calc-silicate rocks (Burack, 1983). These rocks were metamorphosed to upper greenschist facies before the intrusion of the granite (Burack, 1983). The intrusion of the granite into the quartzite and quartzitic schist unit locally resulted in the development of biotite porphyroblasts that transect the metamorphic foliation, the resorption of garnet, and the development of a granoblastic texture. Based upon these changes, Burack (1983) concluded that the contact metamorphism took place at between 475 and $515{ }^{\circ} \mathrm{C}$ and at pressures less than $2 \mathrm{kbar}$, or at a depth of about $6 \mathrm{~km}$.

Granite crops out over an area of only about 2 $\mathrm{km}^{2}$; however, the distribution of contactmetamorphosed rocks suggests that granite underlies much of the Table Mountain area at relatively shallow depths (Burack, 1983). Two phases of the granite are exposed: coarse-grained equigranular biotite granite, and porphyritic biotite granite with a fine-grained groundmass. The coarse-grained granite (Streckeisen, 1975) is composed of 23 percent plagioclase feldspar, 29 percent quartz, 41 percent potassium feldspar, and 7 percent biotite. Plagioclase is altered to white mica, and biotite is altered to chlorite. Because the granite is altered, it has not been dated; similar plutons in the northwest part of the Circle quadrangle have yielded ages of 66 to 57 m.y. B.P. (Wilson and Shew, 1981).

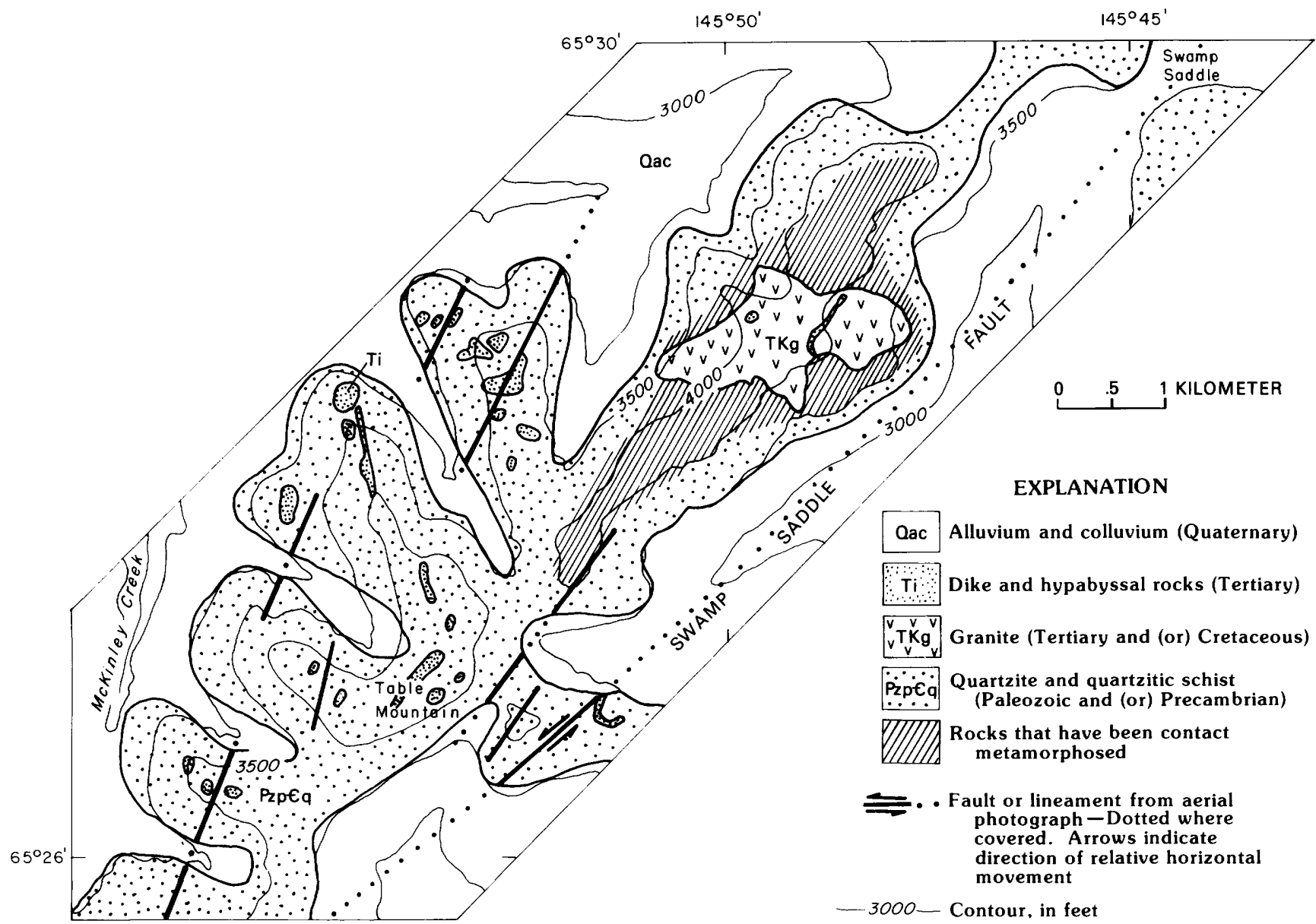

Geology modified from Burack (1983) and Foster and others (1983)

Figure 2. Reconna issance geologic map of Table Mountain area. 
Both the granite and metamorphic rocks have been intruded by dikes and hypabyssal rocks which are most abundant west and southwest of the granite. Most dikes are felsic, but a few are of intermediate composition. Some dikes are aphanitic, others are porphyritic. Phenocrysts in the porphyritic dikes are quartz, potassium feldspar, and plagioclase. The groundmass of the dikes was probably originally plagioclase, potassium feldspar, and quartz, but it is now mainly fine-grained white mica. The quartz phenocrysts have rounded crystal forms suggestive of partial resorption (fig. 3A) and in some cases the quartz phenocrysts are embayed (fig. $3 \underline{B}$ ). Some dikes contain limonite cubes that formed after pyrite(?), and one dike contains minor disseminated purple fluorite in a veinlet. The textures of the dikes suggest that they were emplaced at shallow depths.
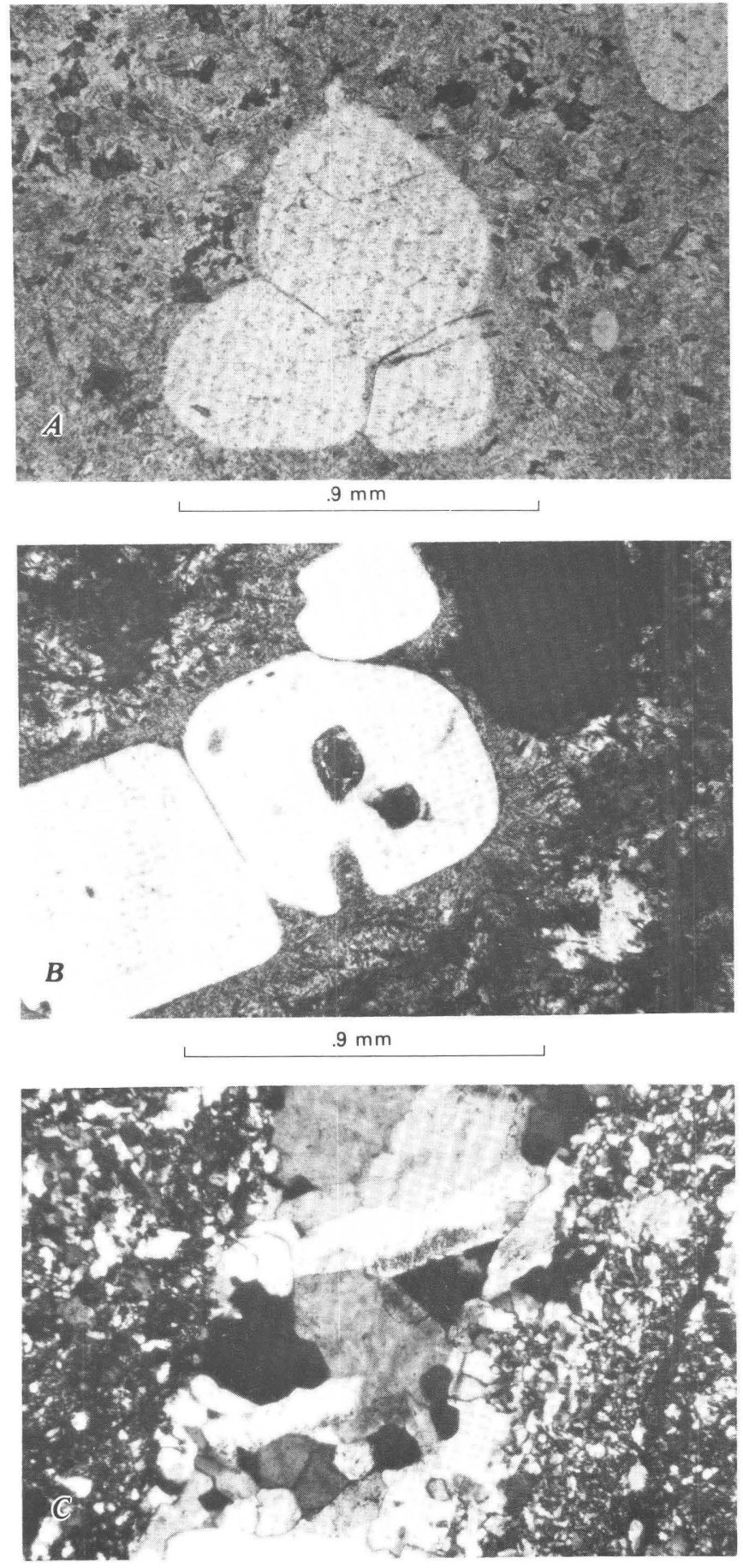

$.9 \mathrm{~mm}$

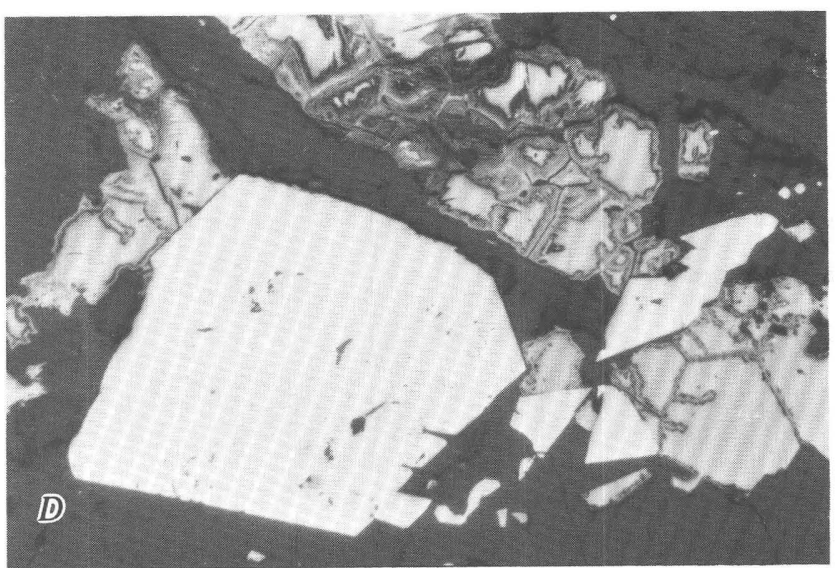

$.05 \mathrm{~mm}$

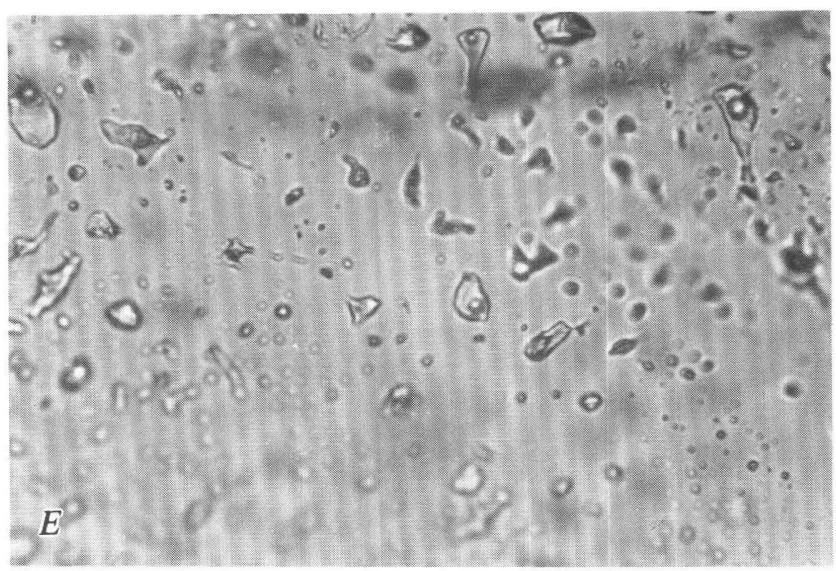

$.05 \mathrm{~mm}$

Figure 3. Photomicrographs of selected thin and polished sections from Table Mountain area. A, Rounded quartz phenocrysts in felsic dike. B, Rounded and embayed quartz phenocryst in felsic dike. C, Quartz veinlet in felsic dike. D, Arsenopyrite surrounded by pyrrhotite from margin of quartz vein. $\underline{E}$, Fluid inclusions from porphyritic felsic dike. 


\section{RESULTS OF INITIAL GEOCHEMICAL SAMPLING}

Table 1 lists analytical data for 5-kg grab samples collected during early phases of the study (Foster and others, 1984). Gold was detected in all of the major rock types present in the area: pelitic and quartzitic metamorphic rocks, calc-silicate rocks, altered granite, and felsic dikes. Figure 4 shows the spatial distribution of the 11 gold-bearing and 72 barren samples. The gold-bearing samples are concentrated in two areas: (1) near Table Mountain and (2) in and adjacent to the exposed granite pluton.

\section{Field Relations of the Gold Occurrences}

\section{Table Mountain Occurrence}

Two samples of tourmaline and sulfide-bearing black biotite schist, which contain 40 and $140 \mathrm{ppm}$ gold, were collected about $3 / 4 \mathrm{~km}$ west of Table Mountain. This area is adjacent to a fault zone, which is characterized by the presence of tourmaline in adjacent rocks (Burack, 1983). During our follow-up study a felsic dike and a sulfide-bearing quartz vein were identified nearby (station 31, fig. 4). The felsic dike rock intrudes schistose quartzite beneath the ridge crest adjacent to the fault. The dike contains phenocrysts of quartz, potassium feldspar, and sericitized plagioclase set in a fine-grained groundmass that has been altered to white mica (fig. 3B,). The dike also contains rare veinlets with disseminated purple fluorite. The margins of the body are brecciated, and the resulting breccia is cut by thin quartz veinlets (fig. $3 \mathrm{C}$ ). In places, breccia fragments are set in a matrix of limonite. Along the ridge, west of the fault zone, a 2.5- to 5-cm-thick arsenopyritebearing quartz vein has been emplaced parallel or subparallel to the foliation of the schistose quartzites. Both the vein and foliation are subhorizontal. The vein contains open spaces and comb structure; arsenopyrite occurs within the vein but is most common as a coating on the outer edges of the vein wall. Tourmaline also occurs locally along the vein wall. We followed the vein for about $100 \mathrm{~m}$ along strike until it was lost in rubble. It seems likely that this 2.5- to 5-cm-thick vein underlies much of the ridge west of the fault-- an area of $0.1 \mathrm{~km}^{2}$.

\section{Occurrences in and Adjacent to the Granite}

Some samples of altered granite and the adjacent quartzite and quartzitic schist contain low levels of gold (see table 1); the gold-bearing samples were found within the pluton and on the north, east, and south sides of the pluton. Two sites were examined more closely, one near the eastern (station 32) and one near the western (station 33) margins of the granite (fig. 4). On the west edge of the granite, a yellow-stained (arsenic?) hypabyssal felsic dike with potassium feldspar, quartz, and biotite phenocrysts in a very fine grained groundmass cuts the equigranular granite. The dike contains fine-grained sulfides, disseminated and as clots in the groundmass. Adjacent to the dike the granite contains clots of probable arsenopyrite. On the east side of the granite, several dikes are exposed that contain large phenocrysts of potassium feldspar, rounded quartz, and altered plagioclase feldspar set in a fine-grained greenish matrix.

\section{Geochemical Results}

Table Mountain Occurrence

Table 2 presents analyses of $5-\mathrm{kg}$ grab samples collected from the Table Mountain occurrences in 1983. The samples were taken from one site, station 31 (fig. 4). Traces of silver and low levels of tin were found in the hypabyssal dike and in an iron-stained sample of the breccia. Two samples of breccia that were not iron stained do not contain silver or tin at detectable levels. Two samples of the quartz vein with sulfides along the vein walls both contained high values of gold, arsenic, and copper. Antimony was detected in both samples.

\section{Occurrences in and Adjacent to the Granite}

Gold was detected only in the sulfide-bearing hypabyssal felsic dike (table 2). Silver was detected in the dike and in the granite adjacent to the dike; tin was also detected in these samples. A sample of this dike without sulfides contained detectable tin but not gold or silver, and a sample of a porphyritic phase of the pluton contained $10 \mathrm{ppm}$ tin but also did not contain detectable gold or silver (table 2). A sample of quartzite from adjacent to the pluton did not contain gold, silver, or tin in detectable amounts. A sample of a dike from the eastern side of the pluton was geochemically indistinct.

\section{Petrography of Mineralized Samples}

\section{Table Mountain Occurrence}

Burack (1983) examined thin sections and a polished section of the gold-bearing black biotite schist in transmitted and reflected light. She reported that they contain abundant biotite, tourmaline, and silver-colored sulfides including arsenopyrite. She also noted that the sulfides cross the weak foliation in the rock and therefore formed after the foliation.

We examined both thin and polished sections of the quartz vein with sulfide-coated outer walls. Sulfides occur within the vein and as a coating with biotite and minor white mica on the outer vein walls. Sulfide minerals identified in and adjacent to the quartz vein were pyrrhotite and arsenopyrite, minor amounts of chalcopyrite, and rarely enargite and sphlalerite. The pyrrhotite is anhedral and cut by cracks along which it is oxidized. Arsenopyrite is euhedral and occurs as bladed crystals which in places appear as islands within the pyrrhotite (fig. 3D). Chalcopyrite is subhedral and in places contains unreplaced pyrrhotite. The enargite is anhedral. The spalerite contains chalcopyrite. 


\section{Occurrences in and Adjacent to the Granite}

Thin sections of the sulfide-bearing felsic dike and the adjacent sulfide-bearing granite were examined in transmitted light. Sulfides in the felsic dike are very fine grained. They are abundant in the groundmass of the dike and occur both disseminated in the groundmass and in clots. The granite adjacent to the dike contains isolated clots of arsenopyrite on the rims of partially altered plagioclase grains.

\section{Fluid-Inclusion Studies}

Fluid inclusions from the quartz vein at the Table Mountain occurrence and from dikes from the occurrences in or adjacent to the pluton were studied by using a heating-freezing stage. Sites for these samples are shown by an asterisk in figure 4 . Of the 20 doubly polished plates that were examined, 6 contain a sufficient number of inclusions of a size large enough $(>5 \mu \mathrm{m}$ along their maximum dimension) to make the heating-and-freezing measurements. The samples contain two major types of fluid inclusions: liquid-rich inclusions and vapor-rich inclusions, termed type I and type II, respectively. Liquid-rich inclusions are more common than vapor-rich ones in the samples that contain both types. Inclusions of both types occur as isolated entities and are unrelated to visible microfractures. Type I inclusions are distributed randomly throughout the quartz; type II inclusions are round, occur in clusters, and show no evidence of necking to adjoining type I inclusions. Therefore both types of inclusions are interpreted as being primary and (or) pseudosecondary and where both types are present, as having been trapped at the same time. Most fluid inclusions for which measurements were made belong to type I; these inclusions generally have less than 15-volume-percent vapor phase at room temperature. Salinities of the fluid inclusions were determined by using the depression-of-freezing-point method described by Roedder (1962).

\section{Table Mountain Occurrence}

Measurements were made on three samples of the quartz vein from the Table Mountain occurrences. Sample $31 \mathrm{~L}^{\prime}$ is white quartz with abundant sulfides; it contains only liquid-rich inclusions. Sample $31 \mathrm{~J}^{\prime \prime}$ is sulfide-bearing vein material with clear and white quartz that contains



Figure 4. Locations of sample sites (see tables 1 and 2). 
Table 1. Analytical data of rocks from the Table Mountain area

[Analysis of $\mathrm{Au}$ by atomic absorption; all other analyses by six-step semiquantitative emission spectrographic methods. $\mathrm{Fe}, \mathrm{Mg}, \mathrm{Ca}$ and $\mathrm{Ti}$ in percent; all others in parts per million. G, greater than the a mount listed; $\mathrm{L}$, less than the amount listed; N, not detected at a mount listed. Analysts included R.M. O'Leary, G.W. Day, E.F., Cooley, P. Risoli, A. Gruzensky, G. Ito, S.J. Sutley, J. Hurrell, G.D. Hof fman, R.R. Carlson, W. Martin, F.J. Takacs, D.L. Brown. J.A. Domenico, D. Galland, J.M. McDade, A.L. Meir, D.G. Murrey, A.J. Toevus, and S. A. Wilson ]

\begin{tabular}{|c|c|c|c|c|c|c|c|c|c|c|c|c|c|}
\hline $\begin{array}{l}\text { Station } \\
\text { number and } \\
\text { sample letter }\end{array}$ & $\begin{array}{c}\text { Rock } \\
\text { description }\end{array}$ & $\mathrm{Fe}$ & $\mathrm{Mg}$ & $\mathrm{Ca}$ & $\mathrm{Ti}$ & $\mathrm{Mn}$ & $\mathrm{Ag}$ & As & B & $\mathrm{Ba}$ & $\mathrm{Be}$ & $\mathrm{Bi}$ & Co \\
\hline $35 \mathrm{~A}$ & Gray quartzite & 2.0 & 1.00 & 0.50 & 0.200 & 300 & $0.5 \mathrm{~N}$ & $200 \mathrm{~N}$ & 30 & $20 \mathrm{~L}$ & $10 \mathrm{~L}$ & $10 \mathrm{~N}$ & $\overline{15}$ \\
\hline $35 B$ & Chloritic schist & 7.0 & 2.00 & 1.50 & .700 & 700 & $.5 \mathrm{~N}$ & $200 \mathrm{~N}$ & 500 & 500 & 15 & $10 \mathrm{~N}$ & 50 \\
\hline $36 \mathrm{C}$ & Gray quartzite & 1.5 & .20 & .05 & .100 & 200 & $.5 \mathrm{~N}$ & $200 \mathrm{~N}$ & 20 & 150 & 10 & $10 N$ & 7 \\
\hline $37 \mathrm{~A}$ & $\begin{array}{l}\text { Gray quartzite with } \\
\text { sulfides }\end{array}$ & 3.0 & .30 & & 200 & 300 & 5 & $200 \mathrm{~N}$ & & & $10 \mathrm{~L}$ & $10 \mathrm{~N}$ & 10 \\
\hline \multirow[t]{2}{*}{38} & Gray quartzite & & (1) & 0 & .200 & (1) & 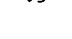 & 2000 & & (30 & & $10 \mathrm{~N}$ & +0 \\
\hline & (iron stained) & 5.0 & 2.00 & 5.00 & .500 & 1,500 & $.5 \mathrm{~L}$ & $200 \mathrm{~N}$ & 10 & 50 & 15 & $10 \mathrm{~N}$ & 30 \\
\hline 39 & Calc-silicate & 2.0 & 5.00 & 10.00 & .050 & 2,000 & $.5 \mathrm{~N}$ & $200 \mathrm{~N}$ & 70 & $20 \mathrm{~N}$ & $10 \mathrm{~L}$ & $10 \mathrm{~N}$ & 5 \\
\hline 40 & $\begin{array}{l}\text { Gray quartzite with } \\
\text { sulfides }\end{array}$ & 5.0 & 2.00 & 2.00 & .300 & 1,000 & $.5 \mathrm{~L}$ & $200 \mathrm{~N}$ & $10 \mathrm{~L}$ & 30 & $10 \mathrm{~L}$ & $10 \mathrm{~N}$ & 30 \\
\hline $42 \mathrm{~A}$ & Granite & 1.5 & 3.00 & .30 & .100 & 300 & $.5 \mathrm{~N}$ & $200 \mathrm{~N}$ & 30 & 500 & 50 & $10 N$ & 5 \\
\hline $43 B$ & $\begin{array}{l}\text { Chloritic schist } \\
\text { (hornfelsed) }\end{array}$ & 5.0 & 10 & & & 500 & $5 \mathrm{t}$ & $200 \mathrm{~N}$ & 70 & 150 & & & 30 \\
\hline $3017 \mathrm{C}$ & Mafic schist & 5.0 & 3.00 & 7.00 & .700 & 1,000 & $.0 \mathrm{~L}$ & $200 \mathrm{~N}$ & $10 \mathrm{~L}$ & 100 & $10 \mathrm{~L}$ & $10 \mathrm{~N}$ & 50 \\
\hline $3020 \mathrm{~A}$ & Dike & 5.0 & 2.00 & .20 & .500 & 500 & $.5 \mathrm{~L}$ & $200 \mathrm{~N}$ & 70 & 300 & 20 & $10 \mathrm{~N}$ & 50 \\
\hline $3075 \mathrm{~A}$ & Gray-brown quartzite & 1.5 & .70 & .70 & .100 & 300 & $.5 \mathrm{~N}$ & $200 \mathrm{~N}$ & 50 & 700 & 20 & $10 N$ & 5 \\
\hline $3075 \mathrm{C}$ & Dike with sulfides & 2.0 & 1.00 & .30 & .150 & 300 & .5 & $200 \mathrm{~N}$ & 30 & 1,500 & 30 & $10 \mathrm{~N}$ & 10 \\
\hline \multirow[t]{2}{*}{$3075 G$} & Quartzite & & & & & & & & & & & & \\
\hline & (iron stained) & 1.5 & .07 & $.05 \mathrm{~L}$ & .050 & 100 & $.5 \mathrm{~L}$ & $200 \mathrm{~N}$ & 20 & 70 & 10 & $10 \mathrm{~N}$ & 15 \\
\hline $3075 \mathrm{~J}$ & Chloritic schist & 3.0 & 1.00 & .07 & .300 & 300 & $.5 \mathrm{~N}$ & $200 \mathrm{~N}$ & 100 & 700 & 30 & $10 \mathrm{~N}$ & 7 \\
\hline $3075 \mathrm{~L}$ & Gray quartzite & 1.5 & .20 & .07 & .070 & 200 & $.5 \mathrm{~L}$ & $200 \mathrm{~N}$ & 50 & 100 & $10 \mathrm{~L}$ & $10 \mathrm{~N}$ & 50 \\
\hline $3075 \mathrm{~N}$ & Gray quartzite & 1.0 & .15 & .50 & .070 & 300 & $.5 \mathrm{~L}$ & $200 \mathrm{~N}$ & 30 & 500 & $10 \mathrm{~L}$ & $10 N$ & 7 \\
\hline \multirow[t]{2}{*}{$3082 B$} & Felsic dike with & & & & & & & & & & & & \\
\hline & $\begin{array}{l}\text { sulfides } \\
\text { Felsic dike }\end{array}$ & 1.0 & .10 & .15 & .030 & 200 & 1.0 & $200 \mathrm{~N}$ & 30 & 150 & 70 & $10 \mathrm{~L}$ & 5 \\
\hline $3082 \mathrm{D}$ & (iron stained) & 1.0 & .07 & $.05 \mathrm{~L}$ & .020 & 200 & 1.5 & $200 \mathrm{~N}$ & 30 & 150 & 30 & $10 \mathrm{~L}$ & 5 \\
\hline $3084 \mathrm{D}$ & Granite & 1.5 & .30 & .20 & .100 & 300 & $.5 \mathrm{~N}$ & $200 \mathrm{~N}$ & 70 & 300 & 150 & $10 \mathrm{~N}$ & 7 \\
\hline $3089 \mathrm{~A}$ & Calcareous quartzite & & & & & & & & & & & & \\
\hline & with sulfides & .7 & .15 & 3.00 & .070 & 300 & $.5 \mathrm{~L}$ & $200 \mathrm{~N}$ & 20 & 300 & 10 & $10 \mathrm{~N}$ & 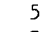 \\
\hline $3090 \mathrm{~B}$ & Calc-silicate & 1.0 & .30 & .50 & .100 & 200 & $.5 \mathrm{~L}$ & $200 \mathrm{~N}$ & 30 & 150 & 10 & $10 \mathrm{~N}$ & 7 \\
\hline $3090 \mathrm{E}$ & Quartzite with & & & & & & & & & & & & \\
\hline & sulfides & 1.5 & .50 & .70 & .150 & 500 & $.5 \mathrm{~L}$ & $200 \mathrm{~N}$ & 15 & 200 & 10 & $10 \mathrm{~N}$ & 30 \\
\hline $3092 B$ & Mafic schist & 5.0 & 5.00 & 7.00 & .700 & 1,500 & .5 & $200 \mathrm{~N}$ & 10 & 150 & $10 \mathrm{~L}$ & $10 \mathrm{~N}$ & 50 \\
\hline $3093 \mathrm{~A}$ & Calc-silicate with & & & & & & & & & & & & \\
\hline & sulfides & 5.0 & 2.00 & .50 & .500 & 700 & $.5 \mathrm{~L}$ & $200 \mathrm{~N}$ & 70 & 300 & 15 & $10 \mathrm{~N}$ & 50 \\
\hline $3095 \mathrm{~A}$ & Black biotite schist & & & & & & & & & & & & \\
\hline & with sulfides & 5.0 & 1.50 & 1.00 & .500 & 500 & 2.0 & $10,000 \mathrm{G}$ & 200 & 300 & 20 & 15 & 50 \\
\hline $3103 \mathrm{~A}$ & Felsic dike & 1.0 & .10 & .15 & .020 & 200 & $.5 \mathrm{~L}$ & 700 & 70 & 300 & 150 & $10 \mathrm{~N}$ & 5 \\
\hline $3106 \mathrm{~A}$ & Chloritic schist & 5.0 & 3.00 & 7.00 & .500 & 1,500 & $.5 \mathrm{~L}$ & $200 \mathrm{~N}$ & 15 & 100 & 15 & $10 \mathrm{~N}$ & 50 \\
\hline $3107 \mathrm{~A}$ & Black biotite schist & 5.0 & 2.00 & .15 & .700 & 200 & 1.0 & $200 \mathrm{~N}$ & $2,000 \mathrm{G}$ & 70 & 50 & $10 \mathrm{~N}$ & 30 \\
\hline $3107 \mathrm{~B}$ & Gray quartzite & 1.5 & .70 & 1.50 & .200 & 500 & $.5 \mathrm{~N}$ & $200 \mathrm{~N}$ & 300 & 300 & 20 & $10 \mathrm{~N}$ & 20 \\
\hline $3107 \mathrm{I}$ & Quartz vein with & & & & & & & & & & & & \\
\hline & sulfides & 5.0 & 5.00 & 10.00 & 1.000 & 5,000 & $0.5 \mathrm{~L}$ & $200 \mathrm{~N}$ & 20 & 500 & 30 & $10 \mathrm{~N}$ & 30 \\
\hline $3107 \mathrm{~L}$ & Dark gray-green & & & & & & & & & & & & \\
\hline & quartzite with sulfides & 10.0 & 7.00 & 10.00 & 1.000 & 2,000 & $.5 \mathrm{~L}$ & $200 \mathrm{~N}$ & 10 & 300 & 10 & $10 \mathrm{~N}$ & 70 \\
\hline $3111 \mathrm{~B}$ & Calc-silicate with & & & & & & & & & & & & \\
\hline & sulfides & 1.5 & 10.00 & 15.00 & .050 & 1,000 & $.5 \mathrm{~N}$ & $200 \mathrm{~N}$ & 100 & $20 \mathrm{~L}$ & $1.0 \mathrm{~L}$ & 20 & 5 \\
\hline $3121 \mathrm{~A}$ & Black biotite schist & 7.0 & 3.00 & 5.00 & 1.000 & 1,500 & $.5 \mathrm{~L}$ & $200 \mathrm{~N}$ & 20 & 50 & $1.0 \mathrm{~L}$ & $10 \mathrm{~N}$ & 30 \\
\hline $3121 \mathrm{D}$ & Felsic dike & .7 & .20 & .15 & .015 & 150 & 1.5 & $200 \mathrm{~N}$ & 70 & 30 & 1.5 & $10 \mathrm{~N}$ & 5 \\
\hline $3121 \mathrm{~F}$ & Gray quartzite & 1.0 & .20 & .15 & .100 & 200 & $.5 \mathrm{~N}$ & $200 \mathrm{~N}$ & $10 \mathrm{~N}$ & 200 & $1.0 \mathrm{~L}$ & $10 \mathrm{~N}$ & 7 \\
\hline $3121 \mathrm{G}$ & Calc-silicate with & & & & & & & & & & & & \\
\hline & sulfides & 10.0 & 1.00 & 10.00 & .070 & $5,000 \mathrm{G}$ & $.5 \mathrm{~L}$ & $200 \mathrm{~N}$ & 10 & 50 & 10.0 & 15 & 30 \\
\hline $3122 \mathrm{~B}$ & Black biotite schist & & & & & & & & & & & & \\
\hline & with sulfides & 5.0 & 1.50 & 2.00 & .700 & 300 & $1.5 \mathrm{~L}$ & 10,000 & 1,500 & 300 & 1.5 & 50 & 70 \\
\hline $3124 \mathrm{~F}$ & Black biotite schist & 3.0 & 1.00 & .15 & 1.000 & 500 & $.5 \mathrm{~N}$ & $200 \mathrm{~N}$ & 100 & 3,000 & 1.5 & $10 \mathrm{~N}$ & 20 \\
\hline $3125 B$ & Blue-gray quartzite & 5.0 & 2.00 & 2.00 & .700 & 2,000 & $.5 \mathrm{~N}$ & $200 \mathrm{~N}$ & 20 & 100 & $1.0 \mathrm{~L}$ & $10 \mathrm{~L}$ & 30 \\
\hline $3125 \mathrm{C}$ & Granite & 1.5 & .30 & .20 & .070 & 200 & .5 & $200 \mathrm{~N}$ & 30 & 1,500 & 3.0 & 15 & 5 \\
\hline $3126 \mathrm{E}$ & Black biotite schist & & & & & & & & & & & & \\
\hline & with sulfides & 5.0 & 1.50 & 3.00 & 1.000 & 1,500 & $.5 \mathrm{~L}$ & $200 \mathrm{~N}$ & 15 & 150 & 1.0 & 20 & 30 \\
\hline $3127 G$ & Gray quartzite & 3.0 & 1.50 & .07 & .500 & 500 & $.5 \mathrm{~N}$ & $200 \mathrm{~N}$ & 70 & 1,000 & 2.0 & $10 \mathrm{~L}$ & 30 \\
\hline $3128 \mathrm{~B}$ & Black biotite schist & 1.5 & .50 & .30 & .100 & 300 & $.5 \mathrm{~L}$ & $200 \mathrm{~N}$ & 150 & 300 & 7.0 & $10 \mathrm{~N}$ & 7 \\
\hline $3129 \mathrm{~A}$ & White quartzite & .7 & .30 & .20 & .200 & 300 & $.5 \mathrm{~N}$ & $200 \mathrm{~N}$ & 20 & 300 & 1.0 & $10 \mathrm{~N}$ & \\
\hline $3129 E$ & Quartz veins & & & & & & & & & & & & \\
\hline & (breccia?) & 1.5 & .30 & $.05 \mathrm{~L}$ & .150 & 300 & $.5 \mathrm{~N}$ & $200 \mathrm{~N}$ & 15 & 300 & 1.0 & $10 \mathrm{~N}$ & 7 \\
\hline $3129 \mathrm{G}$ & Black biotite schist & 2.0 & .70 & .20 & .150 & 300 & $.5 \mathrm{~L}$ & $200 \mathrm{~N}$ & 20 & 200 & 1.0 & $10 \mathrm{~L}$ & 7 \\
\hline $3132 \mathrm{~A}$ & Gray quartzite with & & & & & & & & & & & & \\
\hline & tourmaline & 2.0 & .50 & .15 & .200 & 500 & 1.0 & $200 \mathrm{~N}$ & 2,000 & 500 & 2.0 & $10 \mathrm{~L}$ & 10 \\
\hline $3133 \mathrm{~F}$ & Gray quartzite & 1.5 & .30 & .15 & .150 & 300 & $.5 \mathrm{~N}$ & $200 \mathrm{~N}$ & 30 & 300 & 1.0 & $10 \mathrm{~N}$ & 7 \\
\hline $3135 \mathrm{~A}$ & Black biotite schist & 5.0 & 3.00 & .30 & .700 & 500 & $.5 \mathrm{~L}$ & 200 & 150 & 1,000 & 2.0 & $10 \mathrm{~N}$ & 50 \\
\hline $3136 \mathrm{~A}$ & Calc-silicate with & & & & & & & & & & & & \\
\hline & sulfides & 10.0 & 5.00 & 10.00 & .100 & $5,000 \mathrm{G}$ & $.5 \mathrm{~N}$ & $200 \mathrm{~N}$ & $10 \mathrm{~L}$ & 20 & 3.0 & $10 \mathrm{~N}$ & 30 \\
\hline $3136 \mathrm{~B}$ & Calc-silicate with & & & & & & & & & & & & \\
\hline & sulfides & 7.0 & 3.00 & 10.00 & .150 & $5,000 \mathrm{G}$ & $.5 \mathrm{~N}$ & $200 \mathrm{~N}$ & $10 \mathrm{~L}$ & 100 & 3.0 & $10 \mathrm{~N}$ & 20 \\
\hline
\end{tabular}




\begin{tabular}{|c|c|c|c|c|c|c|c|c|c|c|c|c|c|c|c|c|}
\hline $\mathrm{Cr}$ & $\mathrm{Cu}$ & $\mathbf{L a}$ & Mo & $\mathrm{Nb}$ & $\mathrm{Ni}$ & $\mathrm{Pb}$ & $\mathrm{Sb}$ & $\mathrm{Sc}$ & $\mathrm{Sn}$ & $\mathrm{Sr}$ & $\mathrm{V}$ & W & $\mathrm{Y}$ & $\mathrm{Zn}$ & $\mathrm{Zr}$ & $\overline{\mathrm{Au}}$ \\
\hline 100 & 20 & $20 \mathrm{~L}$ & $5 \mathrm{~N}$ & $20 \mathrm{~N}$ & 30 & $10 \mathrm{~N}$ & $100 \mathrm{~N}$ & 20 & $10 \mathrm{~N}$ & $100 \mathrm{~L}$ & 150 & $50 \mathrm{~N}$ & 20 & $200 \mathrm{~N}$ & 150 & $0.05 \mathrm{~N}$ \\
\hline 500 & 200 & 70 & $5 \mathrm{~N}$ & $20 \mathrm{~L}$ & 70 & 30 & $100 \mathrm{~N}$ & 70 & 10 & 300 & 300 & $50 \mathrm{~L}$ & 50 & $200 \mathrm{~N}$ & 150 & $.05 \mathrm{~N}$ \\
\hline 30 & 20 & 20 & $5 \mathrm{~N}$ & $20 \mathrm{~N}$ & 20 & $10 \mathrm{~N}$ & $100 \mathrm{~N}$ & 5 & $10 \mathrm{~N}$ & $100 \mathrm{~L}$ & 50 & $50 \mathrm{~N}$ & $10 \mathrm{~L}$ & $200 \mathrm{~N}$ & 150 & $.05 \mathrm{~N}$ \\
\hline 70 & 300 & 20 & $5 \mathrm{~N}$ & $20 \mathrm{~N}$ & 30 & $10 \mathrm{~N}$ & $100 \mathrm{~N}$ & 20 & $10 \mathrm{~N}$ & 100 & 100 & $50 \mathrm{~N}$ & 20 & $200 \mathrm{~N}$ & 100 & $.05 \mathrm{~N}$ \\
\hline 50 & 300 & $20 \mathrm{~L}$ & $5 \mathrm{~N}$ & $20 \mathrm{~N}$ & 30 & $10 \mathrm{~N}$ & $100 \mathrm{~N}$ & 50 & 50 & 300 & 200 & $50 \mathrm{~N}$ & 30 & $200 \mathrm{~N}$ & 70 & $.05 \mathrm{~N}$ \\
\hline 15 & 15 & $20 \mathrm{~L}$ & $5 \mathrm{~N}$ & $20 \mathrm{~N}$ & 5 & $10 \mathrm{~L}$ & $100 \mathrm{~N}$ & $5 \mathrm{~L}$ & $10 \mathrm{~N}$ & 100 & 50 & $50 \mathrm{~L}$ & $10 \mathrm{~L}$ & $200 \mathrm{~N}$ & $10 \mathrm{~N}$ & $.05 \mathrm{~N}$ \\
\hline 200 & 50 & $20 \mathrm{~N}$ & $5 \mathrm{~N}$ & $20 \mathrm{~N}$ & 30 & $10 \mathrm{~L}$ & $100 \mathrm{~N}$ & 70 & $10 \mathrm{~N}$ & $100 \mathrm{~N}$ & 200 & $50 \mathrm{~N}$ & 20 & $200 \mathrm{~N}$ & 70 & $.05 \mathrm{~N}$ \\
\hline 20 & 7 & 30 & $5 \mathrm{~N}$ & $20 \mathrm{~L}$ & 10 & 20 & $100 \mathrm{~N}$ & 7 & $10 \mathrm{~N}$ & 200 & 50 & $50 \mathrm{~N}$ & 15 & $200 \mathrm{~N}$ & 100 & $.05 \mathrm{~N}$ \\
\hline 150 & 30 & $20 \mathrm{~L}$ & $5 \mathrm{~N}$ & $20 \mathrm{~N}$ & 50 & 10 & $100 \mathrm{~N}$ & 30 & $10 \mathrm{~N}$ & $100 \mathrm{~N}$ & 200 & $50 \mathrm{~N}$ & 20 & $200 \mathrm{~N}$ & 150 & $.05 \mathrm{~N}$ \\
\hline 100 & 100 & 20 & $5 \mathrm{~N}$ & $20 \mathrm{~N}$ & 70 & $10 \mathrm{~L}$ & $100 \mathrm{~N}$ & 100 & $10 \mathrm{~L}$ & 300 & 500 & $50 \mathrm{~N}$ & 50 & $200 \mathrm{~L}$ & 70 & $.05 \mathrm{~N}$ \\
\hline 300 & 200 & 70 & $5 \mathrm{~N}$ & $20 \mathrm{~L}$ & 70 & 30 & $100 \mathrm{~N}$ & 70 & $10 \mathrm{~N}$ & 100 & 300 & $50 \mathrm{~N}$ & 50 & $200 \mathrm{~L}$ & 150 & $.05 \mathrm{~N}$ \\
\hline 70 & 5 & 100 & $5 \mathrm{~N}$ & $20 \mathrm{~L}$ & 30 & $10 \mathrm{~N}$ & $100 \mathrm{~N}$ & 10 & $10 \mathrm{~N}$ & 150 & 100 & $50 \mathrm{~N}$ & 20 & $200 \mathrm{~N}$ & 150 & $.05 \mathrm{~N}$ \\
\hline 100 & 20 & 100 & $5 \mathrm{~N}$ & 20 & 30 & 50 & $100 \mathrm{~N}$ & 15 & $10 \mathrm{~N}$ & 500 & 150 & $50 \mathrm{~N}$ & 30 & $200 \mathrm{~L}$ & 200 & $.05 \mathrm{~N}$ \\
\hline 10 & 20 & 20 & $5 \mathrm{~N}$ & $20 \mathrm{~N}$ & 50 & $10 \mathrm{~N}$ & $100 \mathrm{~N}$ & $5 \mathrm{~L}$ & $10 \mathrm{~N}$ & $100 \mathrm{~N}$ & $10 \mathrm{~L}$ & $50 \mathrm{~N}$ & 30 & $200 \mathrm{~L}$ & 70 & $.05 \mathrm{~N}$ \\
\hline 200 & 10 & 70 & $5 \mathrm{~N}$ & $20 \mathrm{~L}$ & 20 & 50 & $100 \mathrm{~N}$ & 30 & $10 \mathrm{~N}$ & 150 & 150 & $50 \mathrm{~N}$ & 30 & $200 \mathrm{~L}$ & 100 & $.05 \mathrm{~N}$ \\
\hline 30 & 7 & $20 \mathrm{~L}$ & $5 \mathrm{~N}$ & $20 \mathrm{~N}$ & 70 & $10 \mathrm{~L}$ & $100 \mathrm{~N}$ & 5 & $10 \mathrm{~N}$ & $100 \mathrm{~N}$ & 70 & $50 \mathrm{~N}$ & 15 & $200 \mathrm{~N}$ & 150 & $.05 \mathrm{~N}$ \\
\hline 30 & 5 & 30 & $5 \mathrm{~N}$ & $20 \mathrm{~N}$ & 15 & $10 \mathrm{~N}$ & $100 \mathrm{~N}$ & 5 & $10 \mathrm{~N}$ & 200 & 50 & $50 \mathrm{~N}$ & 15 & $200 \mathrm{~N}$ & 150 & $.05 \mathrm{~N}$ \\
\hline $10 \mathrm{~N}$ & $5 \mathrm{~L}$ & 50 & $5 \mathrm{~N}$ & 30 & 7 & 50 & $100 \mathrm{~N}$ & 5 & 50 & $100 \mathrm{~N}$ & $10 \mathrm{~N}$ & $50 \mathrm{~L}$ & 100 & $200 \mathrm{~N}$ & 100 & $.05 \mathrm{~N}$ \\
\hline $10 \mathrm{~N}$ & $5 \mathrm{~L}$ & 20 & $5 \mathrm{~N}$ & 30 & 5 & 50 & $100 \mathrm{~N}$ & $5 \mathrm{~L}$ & 50 & $100 \mathrm{~N}$ & $10 \mathrm{~N}$ & $50 \mathrm{~N}$ & 70 & $200 \mathrm{~N}$ & 100 & $.05 \mathrm{~N}$ \\
\hline 50 & 20 & 50 & 15 & $20 \mathrm{~L}$ & 30 & $10 \mathrm{~N}$ & $100 \mathrm{~N}$ & 10 & 14 & 150 & 100 & $50 \mathrm{~N}$ & 30 & $200 \mathrm{~N}$ & 100 & $.05 \mathrm{~N}$ \\
\hline 10 & 10 & $20 \mathrm{~N}$ & $5 \mathrm{~N}$ & $20 \mathrm{~N}$ & 10 & $10 \mathrm{~L}$ & $100 \mathrm{~N}$ & $5 \mathrm{~L}$ & $10 \mathrm{~N}$ & 300 & 20 & $50 \mathrm{~N}$ & 10 & $200 \mathrm{~N}$ & 150 & $.05 \mathrm{~N}$ \\
\hline 20 & 15 & 50 & $5 \mathrm{~N}$ & $20 \mathrm{~N}$ & 20 & $10 \mathrm{~N}$ & $100 \mathrm{~N}$ & 5 & $10 \mathrm{~N}$ & 100 & 70 & $50 \mathrm{~N}$ & 20 & $200 \mathrm{~N}$ & 150 & $.05 \mathrm{~N}$ \\
\hline 70 & 50 & 20 & $5 \mathrm{~N}$ & $20 \mathrm{~N}$ & 50 & $10 \mathrm{~N}$ & $100 \mathrm{~N}$ & 15 & $10 \mathrm{~N}$ & 150 & 150 & $50 \mathrm{~N}$ & 20 & $200 \mathrm{~N}$ & 150 & .05 \\
\hline 300 & 150 & $20 \mathrm{~L}$ & 15 & $20 \mathrm{~N}$ & 70 & $10 \mathrm{~L}$ & $100 \mathrm{~N}$ & 70 & 20 & 150 & 500 & $50 \mathrm{~N}$ & 50 & $200 \mathrm{~N}$ & 100 & $.05 \mathrm{~N}$ \\
\hline 300 & 70 & 30 & $5 \mathrm{~N}$ & $20 \mathrm{~L}$ & 70 & 30 & $100 \mathrm{~N}$ & 70 & 20 & 150 & 300 & $50 \mathrm{~N}$ & 30 & 200 & 150 & $.05 \mathrm{~N}$ \\
\hline 150 & 300 & 50 & $5 N$ & $20 \mathrm{~L}$ & 70 & 10 & $100 \mathrm{~N}$ & 50 & $10 \mathrm{~N}$ & 300 & 200 & 50 & 30 & $200 \mathrm{~L}$ & 150 & 140.00 \\
\hline $10 \mathrm{~N}$ & 7 & 20 & $5 \mathrm{~N}$ & 30 & 7 & 30 & $100 \mathrm{~N}$ & $5 \mathrm{~L}$ & 50 & $100 \mathrm{~N}$ & 15 & $50 \mathrm{~N}$ & 70 & $200 \mathrm{~N}$ & 100 & .25 \\
\hline 70 & 500 & 20 & $5 N$ & $20 N$ & 50 & $10 \mathrm{~L}$ & $100 \mathrm{~N}$ & 70 & 70 & 300 & 500 & $50 \mathrm{~N}$ & 30 & $200 \mathrm{~N}$ & 70 & $.05 \mathrm{~N}$ \\
\hline 300 & 50 & 200 & $5 \mathrm{~N}$ & $20 \mathrm{~L}$ & 70 & 70 & $100 \mathrm{~N}$ & 70 & 70 & 50 & 300 & $50 \mathrm{~L}$ & 100 & $200 \mathrm{~L}$ & 150 & $.05 \mathrm{~N}$ \\
\hline 100 & 10 & 50 & $5 \mathrm{~N}$ & $20 \mathrm{~N}$ & 50 & $10 \mathrm{~L}$ & $100 \mathrm{~N}$ & 30 & $10 \mathrm{~N}$ & 300 & 150 & $50 \mathrm{~N}$ & 30 & $200 \mathrm{~N}$ & 150 & $.05 \mathrm{~N}$ \\
\hline 50 & 300 & 30 & $5 \mathrm{~N}$ & $20 \mathrm{~N}$ & 50 & $10 \mathrm{~L}$ & $100 \mathrm{~N}$ & 50 & 30 & 700 & 200 & $50 \mathrm{~N}$ & 30 & $200 \mathrm{~N}$ & 70 & $.05 \mathrm{~N}$ \\
\hline 70 & 70 & 20 & $5 \mathrm{~N}$ & $20 \mathrm{~N}$ & 70 & 15 & $100 \mathrm{~N}$ & 50 & 20 & 200 & 300 & $50 \mathrm{~N}$ & 50 & $200 \mathrm{~N}$ & 100 & $.05 \mathrm{~N}$ \\
\hline 20 & 7 & $20 \mathrm{~L}$ & $5 \mathrm{~N}$ & $20 \mathrm{~N}$ & 15 & $10 \mathrm{~L}$ & $100 \mathrm{~N}$ & 5 & 30 & 300 & 100 & 70 & 10 & $200 \mathrm{~N}$ & 15 & $.05 \mathrm{~N}$ \\
\hline 500 & 700 & 50 & $5 N$ & $20 \mathrm{~L}$ & 50 & $10 \mathrm{~L}$ & $100 \mathrm{~N}$ & 70 & 30 & 300 & 300 & $50 \mathrm{~L}$ & 30 & $200 \mathrm{~L}$ & 200 & $.05 \mathrm{~N}$ \\
\hline $10 \mathrm{~N}$ & 5 & 50 & $5 \mathrm{~N}$ & 30 & 5 & 50 & $100 \mathrm{~N}$ & $5 \mathrm{~N}$ & 50 & $100 \mathrm{~N}$ & $10 \mathrm{~N}$ & $50 \mathrm{~N}$ & 100 & $200 \mathrm{~N}$ & 100 & $.05 \mathrm{~N}$ \\
\hline 20 & 5 & 30 & $5 N$ & $20 \mathrm{~N}$ & 10 & $10 \mathrm{~N}$ & $100 \mathrm{~N}$ & $5 \mathrm{~L}$ & $10 \mathrm{~N}$ & $100 \mathrm{~L}$ & 70 & $50 \mathrm{~N}$ & 10 & $200 \mathrm{~N}$ & 200 & $.05 \mathrm{~N}$ \\
\hline 70 & 150 & $20 \mathrm{~L}$ & $5 N$ & $20 \mathrm{~N}$ & 30 & $10 \mathrm{~N}$ & $100 \mathrm{~N}$ & 50 & 100 & 100 & 200 & 150 & 50 & 300 & 100 & $.05 \mathrm{~N}$ \\
\hline 300 & 300 & 100 & $5 \mathrm{~N}$ & $20 \mathrm{~L}$ & 70 & 20 & $100 \mathrm{~N}$ & 70 & 30 & 300 & 300 & 50 & 70 & $200 \mathrm{~N}$ & 150 & 40.00 \\
\hline 500 & 100 & 150 & $5 \mathrm{~N}$ & $20 \mathrm{~L}$ & 70 & 30 & $100 \mathrm{~N}$ & 70 & $10 \mathrm{~N}$ & 300 & 500 & $50 \mathrm{~N}$ & 100 & $200 \mathrm{~N}$ & 300 & .05 \\
\hline 300 & 150 & 20 & $5 N$ & $20 \mathrm{~N}$ & 70 & $10 \mathrm{~L}$ & $100 \mathrm{~N}$ & 50 & 30 & 100 & 500 & $50 \mathrm{~N}$ & 30 & $200 \mathrm{~N}$ & 200 & $.05 \mathrm{~N}$ \\
\hline 30 & 15 & 100 & 200 & $20 \mathrm{~L}$ & 10 & 70 & $100 \mathrm{~N}$ & 5 & $10 \mathrm{~N}$ & 200 & 70 & $50 \mathrm{~N}$ & 20 & 500 & 70 & $.05 \mathrm{~N}$ \\
\hline 500 & 200 & 50 & $5 \mathrm{~N}$ & $20 \mathrm{~N}$ & 70 & $10 \mathrm{~N}$ & $100 \mathrm{~N}$ & 70 & 30 & 100 & 300 & $50 \mathrm{~N}$ & 30 & $200 \mathrm{~N}$ & 200 & .10 \\
\hline 300 & 100 & 70 & $5 \mathrm{~N}$ & $20 \mathrm{~L}$ & 70 & 30 & $100 \mathrm{~N}$ & 50 & $10 \mathrm{~N}$ & $100 \mathrm{~L}$ & 200 & $50 \mathrm{~N}$ & 50 & $200 \mathrm{~N}$ & 150 & $.05 \mathrm{~N}$ \\
\hline 70 & 7 & 70 & 5 & $20 \mathrm{~N}$ & 30 & 20 & $100 \mathrm{~N}$ & 10 & $10 \mathrm{~N}$ & 150 & 150 & $50 \mathrm{~N}$ & 15 & $200 \mathrm{~N}$ & 150 & $.05 \mathrm{~N}$ \\
\hline 20 & 7 & $20 \mathrm{~N}$ & $5 \mathrm{~N}$ & $20 \mathrm{~N}$ & 7 & $10 \mathrm{~N}$ & $100 \mathrm{~N}$ & 5 & $10 \mathrm{~N}$ & $100 \mathrm{~L}$ & 20 & $50 \mathrm{~N}$ & 10 & $200 \mathrm{~N}$ & 200 & $.05 \mathrm{~N}$ \\
\hline 30 & $5 \mathrm{~L}$ & $20 \mathrm{~L}$ & $5 \mathrm{~N}$ & $20 \mathrm{~N}$ & 30 & $10 \mathrm{~N}$ & $100 \mathrm{~N}$ & 70 & $10 \mathrm{~N}$ & $100 \mathrm{~N}$ & 70 & $50 \mathrm{~L}$ & 20 & $200 \mathrm{~N}$ & 200 & $.05 \mathrm{~N}$ \\
\hline 70 & 700 & 20 & $5 \mathrm{~N}$ & $20 \mathrm{~N}$ & 15 & $10 \mathrm{~N}$ & $100 \mathrm{~N}$ & 15 & $10 \mathrm{~N}$ & $100 \mathrm{~L}$ & 150 & $50 \mathrm{~N}$ & 20 & $200 \mathrm{~N}$ & 100 & $.05 \mathrm{~N}$ \\
\hline 50 & 50 & 50 & $5 \mathrm{~N}$ & $20 \mathrm{~N}$ & 15 & 20 & $100 \mathrm{~N}$ & 7 & $10 \mathrm{~N}$ & $100 \mathrm{~L}$ & 70 & $50 \mathrm{~N}$ & 20 & $200 \mathrm{~N}$ & 500 & $.05 \mathrm{~N}$ \\
\hline 50 & 10 & 30 & $5 \mathrm{~N}$ & $20 \mathrm{~N}$ & 20 & 10 & $100 \mathrm{~N}$ & 10 & $10 \mathrm{~N}$ & 100 & 70 & $50 \mathrm{~N}$ & 20 & $200 \mathrm{~N}$ & 150 & $.05 \mathrm{~N}$ \\
\hline 300 & 500 & 70 & $5 \mathrm{~N}$ & $20 \mathrm{~L}$ & 70 & 30 & $100 \mathrm{~N}$ & 70 & $10 \mathrm{~N}$ & $100 \mathrm{~L}$ & 300 & $50 \mathrm{~N}$ & 70 & $200 \mathrm{~L}$ & 150 & $.05 \mathrm{~N}$ \\
\hline 70 & 70 & 50 & $5 \mathrm{~N}$ & $20 \mathrm{~L}$ & 50 & $10 \mathrm{~N}$ & $100 \mathrm{~N}$ & 15 & 100 & 100 & 100 & 500 & 30 & 500 & 100 & $.05 \mathrm{~N}$ \\
\hline 200 & 30 & 100 & $5 \mathrm{~N}$ & 20 & 30 & $10 \mathrm{~N}$ & $100 \mathrm{~N}$ & 30 & 70 & 700 & 150 & 50 & 50 & 300 & 150 & $.05 \mathrm{~N}$ \\
\hline
\end{tabular}


Table 1. Analytical data of rocks from the Table Mountain area-Continued

\begin{tabular}{|c|c|c|c|c|c|c|c|c|c|c|c|c|c|}
\hline $\begin{array}{l}\text { Station } \\
\text { number and } \\
\text { sample letter }\end{array}$ & $\begin{array}{c}\text { Rock } \\
\text { description }\end{array}$ & $\mathrm{Fe}$ & $\mathrm{Mg}$ & $\mathrm{Ca}$ & $\mathrm{Ti}$ & $\mathrm{Mn}$ & $\mathrm{Ag}$ & As & $\mathrm{B}$ & $\overline{\mathrm{Ba}}$ & $\mathrm{Be}$ & $\mathrm{Bi}$ & $\overline{\mathrm{Co}}$ \\
\hline $3136 \mathrm{C}$ & $\begin{array}{l}\text { Calc-silicate with } \\
\text { sulfides }\end{array}$ & 10.0 & 5.00 & 10.00 & 0.100 & $5,000 \mathrm{G}$ & $0.5 \mathrm{~N}$ & $200 \mathrm{~N}$ & $10 \mathrm{~L}$ & $20 \mathrm{~N}$ & 5.0 & $10 \mathrm{~N}$ & 30 \\
\hline $3136 \mathrm{~F}$ & Calc-silicate & 5.0 & 1.50 & 15.00 & .100 & 5,000 & $.5 \mathrm{~N}$ & $200 \mathrm{~N}$ & $10 \mathrm{~L}$ & 70 & 1.5 & $10 \mathrm{~N}$ & 20 \\
\hline $3136 \mathrm{H}$ & Calc-silicate & 1.0 & 1.50 & 20.00 & .070 & 500 & $.5 \mathrm{~N}$ & $200 \mathrm{~N}$ & $10 \mathrm{~N}$ & 100 & $1.0 \mathrm{~L}$ & $10 \mathrm{~N}$ & 5 \\
\hline $3137 \mathrm{D}$ & $\begin{array}{l}\text { Gray quartzite with } \\
\text { sulfides }\end{array}$ & 5.0 & 2.00 & 7.00 & .200 & 3,000 & .5 & $200 \mathrm{~N}$ & 20 & 100 & 1.0 & $10 \mathrm{~N}$ & 30 \\
\hline $\begin{array}{l}3139 C \\
3139 D\end{array}$ & $\begin{array}{l}\text { Breccia } \\
\quad \text { (iron stained) } \\
\text { Black biotite schist }\end{array}$ & 1.5 & .30 & .15 & .070 & 200 & $.5 \mathrm{~L}$ & $200 \mathrm{~N}$ & $10 \mathrm{~N}$ & 150 & 1.5 & $10 \mathrm{~N}$ & 5 \\
\hline $3139 E$ & $\begin{array}{l}\text { with sulfides } \\
\text { Gray quartzite with }\end{array}$ & 7.0 & 3.00 & .70 & .700 & 2,000 & $.5 \mathrm{~L}$ & $200 \mathrm{~N}$ & 150 & 500 & 1.0 & $10 \mathrm{~N}$ & 50 \\
\hline $3139 \mathrm{~J}$ & $\begin{array}{l}\text { sulfides } \\
\text { Gray quartzite with }\end{array}$ & 3.0 & 1.00 & 1.00 & .500 & 700 & $.5 \mathrm{~L}$ & $200 \mathrm{~N}$ & 20 & 70 & $1.0 \mathrm{~L}$ & 20 & 30 \\
\hline & sulfides & 3.0 & 1.00 & 3.00 & .300 & 1,000 & $.5 \mathrm{~N}$ & $200 \mathrm{~N}$ & 15 & 30 & 1.0 & 20 & 7 \\
\hline $3140 \mathrm{I}$ & $\begin{array}{l}\text { Black biotite schist } \\
\text { with quartz vein } \\
\text { and sulfides }\end{array}$ & 5.0 & 2.00 & .20 & .700 & 1,000 & $.5 \mathrm{~L}$ & $200 \mathrm{~N}$ & 70 & 500 & 1.5 & $10 \mathrm{~N}$ & 30 \\
\hline $3145 \mathrm{C}$ & $\begin{array}{l}\text { Gray quartzite with } \\
\text { vein }\end{array}$ & 2.0 & .50 & .10 & .150 & 300 & .5 & $200 \mathrm{~N}$ & 70 & 300 & 1.0 & $10 \mathrm{~N}$ & 20 \\
\hline $3145 \mathrm{E}$ & Black biotite schist & 5.0 & 1.50 & .07 & .700 & 500 & $.5 \mathrm{~L}$ & $200 \mathrm{~N}$ & 100 & 700 & 2.0 & $10 N$ & 30 \\
\hline $3146 \mathrm{D}$ & Gray quartzite & 3.0 & 1.00 & .10 & .500 & 500 & $.5 \mathrm{~L}$ & $200 \mathrm{~N}$ & 30 & 1,000 & 2.0 & $10 \mathrm{~N}$ & 20 \\
\hline $3147 \mathrm{~B}$ & Black biotite schist & 3.0 & 1.50 & .15 & .500 & 700 & $.5 \mathrm{~N}$ & $200 \mathrm{~N}$ & 1,000 & 1,500 & 3.0 & $10 \mathrm{~N}$ & 20 \\
\hline $3148 B$ & Intermediate dike & 1.5 & .70 & .50 & .300 & 300 & $.5 \mathrm{~N}$ & $200 \mathrm{~N}$ & 30 & 1,000 & 2.0 & $10 \mathrm{~N}$ & 10 \\
\hline $3148 \mathrm{C}$ & Intermediate dike & 5.0 & 5.00 & 5.00 & .700 & 700 & $.5 \mathrm{~N}$ & $200 \mathrm{~N}$ & 10 & 1,000 & $1.0 \mathrm{~L}$ & $10 \mathrm{~N}$ & 50 \\
\hline $3148 \mathrm{D}$ & Intermediate dike & 5.0 & 5.00 & 5.00 & .700 & 1,000 & $.5 \mathrm{~L}$ & $200 \mathrm{~N}$ & 10 & 700 & 1.0 & $10 N$ & 30 \\
\hline $3148 \mathrm{E}$ & Intermediate dike & 3.0 & 1.50 & 3.00 & .700 & 500 & .5 & $200 \mathrm{~N}$ & 20 & 1,500 & 3.0 & $10 \mathrm{~N}$ & 20 \\
\hline $3148 \mathrm{~F}$ & Granite & 10.0 & .02 & $.05 \mathrm{~L}$ & .070 & 15 & 2.0 & $10,000 \mathrm{G}$ & $10 \mathrm{~L}$ & 100 & 1.5 & 20 & 30 \\
\hline $3149 B$ & Granite & 1.5 & .30 & .05 & .070 & 200 & 1.0 & 1,000 & 30 & 700 & 2.0 & $10 \mathrm{~N}$ & 5 \\
\hline $3150 \mathrm{D}$ & Quartzite & 1.5 & .50 & 1.00 & .200 & 500 & $.5 \mathrm{~N}$ & 300 & 20 & 70 & $1.0 \mathrm{~L}$. & $10 \mathrm{~N}$ & 10 \\
\hline $3150 G$ & Calc-silicate & 15.0 & 1.50 & 10.00 & .300 & $5,000 \mathrm{G}$ & $.5 \mathrm{~L}$ & 200 & 20 & 100 & 70.0 & 30 & 30 \\
\hline $3150 \mathrm{H}$ & $\begin{array}{l}\text { Calc-silicate with } \\
\text { sulfides }\end{array}$ & 5.0 & 1.50 & 10.00 & .300 & 5,000 & $.5 \mathrm{~L}$ & $200 \mathrm{~N}$ & 15 & $20 \mathrm{~L}$ & 7.0 & $10 \mathrm{~L}$ & 20 \\
\hline $3150 \mathrm{I}$ & $\begin{array}{l}\text { Calc-silicate with } \\
\text { sulfides }\end{array}$ & 7.0 & 1.50 & 15.00 & .700 & $5,000 \mathrm{G}$ & $.5 \mathrm{~L}$ & $200 \mathrm{~N}$ & 10 & 50 & 7.0 & $10 \mathrm{~N}$ & 20 \\
\hline $3150 \mathrm{~K}$ & Calc-silicate & 7.0 & 2.00 & 5.00 & 1.000 & $5,000 \mathrm{G}$ & $.5 \mathrm{~N}$ & $200 \mathrm{~N}$ & $10 \mathrm{~L}$ & 70 & 7.0 & $10 \mathrm{~N}$ & 20 \\
\hline $3151 \mathrm{C}$ & Blue-green quartzite & 1.5 & .30 & .20 & .150 & 300 & $.5 \mathrm{~N}$ & $200 \mathrm{~N}$ & 15 & 100 & 1.0 & $10 \mathrm{~N}$ & 10 \\
\hline $3152 \mathrm{E}$ & Chloritic quartzite & 3.0 & 1.50 & 5.00 & .200 & 1,000 & $.5 \mathrm{~N}$ & $200 \mathrm{~N}$ & 20 & 30 & $1.0 \mathrm{~L}$ & $10 \mathrm{~N}$ & 20 \\
\hline 0044B & Calc-silicate & 10.0 & 1.00 & 15.00 & .500 & $5,000 \mathrm{G}$ & $.5 \mathrm{~N}$ & $200 \mathrm{~N}$ & $10 \mathrm{~L}$ & $20 \mathrm{~L}$ & 5.0 & 50 & 7 \\
\hline $44 \mathrm{~A}$ & Felsic dike & 1.0 & .15 & .05 & .020 & 500 & 1.0 & $200 \mathrm{~N}$ & 15 & 70 & 15.0 & $10 \mathrm{~L}$ & $5 \mathrm{~L}$ \\
\hline $44 \mathrm{~B}$ & Gray quartz & & & & & & & & & & & & \\
\hline & (iron stained) & 3.0 & .70 & 1.00 & .200 & 500 & .5 & $200 \mathrm{~N}$ & 20 & 200 & 1.5 & $10 N$ & 50 \\
\hline $44 \mathrm{C}$ & Calc-silicate with & & & & & & & & & & & & \\
\hline & sulfides & 10.0 & 1.00 & 15.00 & .150 & 5,000 & $.5 \mathrm{~N}$ & $200 \mathrm{~N}$ & 20 & $20 \mathrm{~L}$ & 7.0 & 30 & 15 \\
\hline 44D & $\begin{array}{l}\text { Calc-silicate with } \\
\text { sulfides }\end{array}$ & 5.0 & 3.00 & 10.00 & .700 & 3,000 & $.5 \mathrm{~N}$ & $200 \mathrm{~N}$ & 30 & 100 & 20.0 & $10 L$ & 20 \\
\hline
\end{tabular}

both liquid- and vapor-rich fluid inclusions. Sample $31 \mathrm{I}$ is white and clear quartz from the vein. The sample contains both vapor-rich and liquid-rich fluid inclusions. Salinities of fluid inclusions range from 0 to 9.9-weight-percent $\mathrm{NaCl}$ equivalent; most are from 3 to 7 percent. Heating data are shown separately in histograms (fig. 5).

The histograms for the homogenization temperatures are strongly unimodal for all three samples and the modal temperature of the type I inclusions is approximately equal in all three samples (sample $31 \mathrm{I}, 265{ }^{\circ} \mathrm{C}$; sample $31 \mathrm{~J}^{\prime \prime \prime}, 255^{\circ} \mathrm{C}$; and sample $31 \mathrm{~L}, 275^{\circ} \mathrm{C}$ ). Homogenization temperatures for type II inclusions, in samples $31 \mathrm{I}$ and $31 \mathrm{J' \prime \prime}$, range from 260 to $380^{\circ} \mathrm{C}$--values which coincide with homogenization temperatures for the upper half of type I inclusions (see fig. 5). The majority of the homogenization temperatures for type II inclusions range from 320 to $380{ }^{\circ} \mathrm{C}$. Because type II and some type I inclusions occur together and appear to have formed at the same time, we interpret the data as recording the cooling of an initially boiling aqueous fluid. Boiling appears to have begun at about $370{ }^{\circ} \mathrm{C}$ and continued as the system cooled to about $320{ }^{\circ} \mathrm{C}$. Because type I inclusions are more common in the samples than type II inclusions, it appears that most of the vapor phase was lost during boiling. Most of the type I inclusions were trapped below $320{ }^{\circ} \mathrm{C}$ when the fluid probably was a liquid. The presence in sample $31 \mathrm{I}$ of type II inclusions that have homogenization temperatures below $320{ }^{\circ} \mathrm{C}$ are interpreted as being the result of measurement errors that can result from difficulties in observing the exact temperature at which liquid homogenizes in vapor-rich fluid inclusions (see Bodnar and others, 1985, p. 1867). Assuming that the quartz vein was open to the surface (that is, the pressure was hydrostatic) and the fluid was boiling throughout the fluid column, the temperature-depth relationship of Haas (1971) may be used to calculate the depth of formation of the vein. A fluid with a salinity of 5weight-percent $\mathrm{NaCl}$ and a temperature of 320 to $370{ }^{\circ} \mathrm{C}$ will boil at depths less than 1,400 to $2,400 \mathrm{~m}$. 


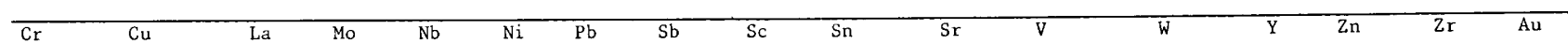

\begin{tabular}{|c|c|c|c|c|c|c|c|c|c|c|c|c|c|c|c|c|}
\hline 150 & 100 & 70 & $5 \mathrm{~N}$ & $20 \mathrm{~L}$ & 50 & $10 \mathrm{~N}$ & $100 \mathrm{~N}$ & 20 & 100 & 100 & 100 & 300 & 50 & 700 & 150 & $0.05 \mathrm{~L}$ \\
\hline 100 & 10 & 70 & $5 \mathrm{~N}$ & $30 \mathrm{~L}$ & 20 & $10 \mathrm{~L}$ & $100 \mathrm{~N}$ & 15 & 150 & 100 & 150 & $50 \mathrm{~L}$ & 30 & 300 & 150 & $.05 \mathrm{~N}$ \\
\hline 30 & 10 & $20 \mathrm{~N}$ & $5 \mathrm{~N}$ & $20 \mathrm{~N}$ & 5 & 20 & $100 \mathrm{~N}$ & SL & $10 \mathrm{~N}$ & 1,000 & 70 & $50 \mathrm{~N}$ & 15 & $200 \mathrm{~N}$ & 70 & $.05 \mathrm{~N}$ \\
\hline 70 & 500 & 30 & $5 \mathrm{~N}$ & $20 \mathrm{~N}$ & 30 & $10 \mathrm{~N}$ & $100 \mathrm{~N}$ & 50 & $10 \mathrm{~N}$ & 300 & 150 & $50 \mathrm{~N}$ & 30 & $200 \mathrm{~N}$ & 100 & $.05 \mathrm{~N}$ \\
\hline 30 & 50 & $20 \mathrm{~N}$ & $5 \mathrm{~N}$ & $20 \mathrm{~N}$ & 15 & $10 \mathrm{~N}$ & $100 \mathrm{~N}$ & 10 & $10 \mathrm{~N}$ & $100 \mathrm{~N}$ & 70 & $50 \mathrm{~L}$ & $10 \mathrm{~N}$ & $200 \mathrm{~N}$ & 50 & $.05 \mathrm{~N}$ \\
\hline 500 & 300 & 70 & $5 \mathrm{~N}$ & $20 \mathrm{~L}$ & 70 & 30 & $100 \mathrm{~N}$ & 70 & $10 \mathrm{~L}$ & $100 \mathrm{~L}$ & 500 & $50 \mathrm{~N}$ & 70 & $200 \mathrm{~L}$ & 200 & $.05 \mathrm{~N}$ \\
\hline 100 & 300 & 20 & $5 \mathrm{~N}$ & $20 \mathrm{~N}$ & 50 & $10 \mathrm{~N}$ & $100 \mathrm{~N}$ & 30 & $10 \mathrm{~N}$ & $100 \mathrm{~L}$ & 200 & $50 \mathrm{~N}$ & 20 & $200 \mathrm{~N}$ & 150 & .05 \\
\hline 100 & 200 & 30 & $5 \mathrm{~N}$ & $20 \mathrm{~N}$ & 30 & $10 \mathrm{~N}$ & $100 \mathrm{~N}$ & 30 & 15 & $100 \mathrm{~L}$ & 150 & $50 \mathrm{~L}$ & 20 & $200 \mathrm{~N}$ & 100 & .20 \\
\hline 300 & 300 & 100 & 10 & $20 \mathrm{~L}$ & 70 & 30 & $100 \mathrm{~N}$ & 70 & $10 \mathrm{~N}$ & $100 \mathrm{~L}$ & 300 & $50 \mathrm{~N}$ & 50 & $200 \mathrm{~L}$ & 200 & $.05 \mathrm{~L}$ \\
\hline 50 & 30 & 20 & $5 \mathrm{~N}$ & $20 \mathrm{~N}$ & 50 & 50 & $100 \mathrm{~N}$ & 15 & $10 \mathrm{~N}$ & $100 \mathrm{~L}$ & 100 & $50 \mathrm{~N}$ & 20 & $200 \mathrm{~L}$ & 150 & $.05 \mathrm{~N}$ \\
\hline 200 & 70 & 70 & $5 \mathrm{~N}$ & $20 \mathrm{~L}$ & 50 & 30 & $100 \mathrm{~N}$ & 50 & $10 \mathrm{~N}$ & $100 \mathrm{~L}$ & 200 & $50 \mathrm{~N}$ & 50 & $200 \mathrm{~L}$ & 200 & $.05 \mathrm{~N}$ \\
\hline 100 & $5 \mathrm{~L}$ & 70 & $5 \mathrm{~N}$ & $20 \mathrm{~L}$ & 50 & 10 & $100 \mathrm{~N}$ & 30 & $10 \mathrm{~N}$ & 150 & 150 & $50 \mathrm{~N}$ & 30 & $200 \mathrm{~N}$ & 200 & $.05 \mathrm{~N}$ \\
\hline 300 & 15 & 150 & $5 \mathrm{~N}$ & $20 \mathrm{~L}$ & 50 & 30 & $100 \mathrm{~N}$ & 50 & 50 & 200 & 200 & $50 \mathrm{~N}$ & 70 & $200 \mathrm{~L}$ & 150 & $.05 \mathrm{~N}$ \\
\hline 150 & 10 & 100 & $5 \mathrm{~N}$ & $20 \mathrm{~L}$ & 5 & 50 & $100 \mathrm{~N}$ & 15 & 10 & 150 & 100 & $50 \mathrm{~N}$ & 50 & $200 \mathrm{~N}$ & 200 & $.05 \mathrm{~N}$ \\
\hline 700 & 20 & 50 & $5 \mathrm{~N}$ & $20 \mathrm{~L}$ & 20 & 15 & $100 \mathrm{~N}$ & 70 & $10 \mathrm{~N}$ & 500 & 200 & $50 \mathrm{~N}$ & 50 & $200 \mathrm{~L}$ & 150 & $.05 \mathrm{~N}$ \\
\hline 700 & 20 & 50 & $5 \mathrm{~N}$ & $20 \mathrm{~L}$ & 15 & 20 & $100 \mathrm{~N}$ & 50 & $10 \mathrm{~N}$ & 300 & 200 & $50 \mathrm{~N}$ & 50 & $200 \mathrm{~L}$ & 150 & $.05 \mathrm{~N}$ \\
\hline 500 & 20 & 100 & $5 \mathrm{~N}$ & $20 \mathrm{~L}$ & 10 & 50 & $100 \mathrm{~N}$ & 30 & $10 \mathrm{~N}$ & 300 & 150 & $50 \mathrm{~N}$ & 50 & $200 \mathrm{~L}$ & 200 & $.05 \mathrm{~N}$ \\
\hline 30 & $5 \mathrm{~L}$ & 20 & $5 \mathrm{~N}$ & $20 \mathrm{~L}$ & $5 \mathrm{~L}$ & 150 & 100 & 10 & 50 & 100 & 50 & $50 \mathrm{~L}$ & 10 & $200 \mathrm{~N}$ & 70 & .20 \\
\hline 30 & 15 & $20 \mathrm{~L}$ & $5 \mathrm{~N}$ & $20 \mathrm{~L}$ & 7 & 70 & $100 \mathrm{~N}$ & 10 & $10 \mathrm{~N}$ & 100 & 70 & $50 \mathrm{~N}$ & 20 & 200 & 70 & $.05 \mathrm{~N}$ \\
\hline 70 & 7 & 20 & $5 \mathrm{~N}$ & $20 \mathrm{~N}$ & 30 & $10 \mathrm{~N}$ & $100 \mathrm{~N}$ & 15 & $10 \mathrm{~N}$ & 100 & 100 & $50 \mathrm{~N}$ & 15 & $200 \mathrm{~N}$ & 100 & $.05 \mathrm{~N}$ \\
\hline 100 & 500 & 50 & $5 \mathrm{~N}$ & $20 \mathrm{~L}$ & 30 & $10 \mathrm{~L}$ & $100 \mathrm{~N}$ & 20 & 150 & 300 & 150 & $50 \mathrm{~N}$ & 30 & 300 & 150 & $.05 \mathrm{~N}$ \\
\hline 200 & 100 & 100 & $5 N$ & $20 \mathrm{~L}$ & 50 & $10 \mathrm{~L}$ & $100 \mathrm{~N}$ & 50 & 150 & 300 & 150 & $50 \mathrm{~N}$ & 30 & 300 & 150 & $.05 \mathrm{~N}$ \\
\hline 300 & 300 & 100 & $5 \mathrm{~N}$ & 20 & 30 & $10 \mathrm{~L}$ & $100 \mathrm{~N}$ & 50 & 150 & 300 & 150 & $50 \mathrm{~N}$ & 50 & 300 & 200 & $.05 \mathrm{~N}$ \\
\hline 200 & 200 & 200 & $5 \mathrm{~N}$ & 50 & 15 & $10 \mathrm{~L}$ & $100 \mathrm{~N}$ & 70 & 150 & $100 \mathrm{~N}$ & 100 & $50 \mathrm{~N}$ & 50 & 200 & 500 & $.05 \mathrm{~N}$ \\
\hline 70 & 20 & 20 & $5 N$ & $20 \mathrm{~N}$ & 30 & $10 \mathrm{~N}$ & $100 \mathrm{~N}$ & 10 & $10 \mathrm{~N}$ & 100 & 100 & $50 \mathrm{~N}$ & 30 & $200 \mathrm{~N}$ & 150 & $.05 \mathrm{~N}$ \\
\hline 200 & $5 \mathrm{~L}$ & $20 \mathrm{~L}$ & $5 \mathrm{~N}$ & $20 \mathrm{~N}$ & 70 & $10 \mathrm{~L}$ & $100 \mathrm{~N}$ & 30 & $10 \mathrm{~N}$ & 100 & 200 & $50 \mathrm{~N}$ & 20 & $200 \mathrm{~N}$ & 150 & $.05 \mathrm{~N}$ \\
\hline 20 & 200 & $20 \mathrm{~N}$ & $5 \mathrm{~N}$ & $20 \mathrm{~N}$ & 20 & $20 \mathrm{~N}$ & $100 \mathrm{~N}$ & $5 \mathrm{~L}$ & 150 & 200 & 200 & $50 \mathrm{~N}$ & 15 & 500 & 100 & $.05 \mathrm{~N}$ \\
\hline $10 \mathrm{~L}$ & $5 \mathrm{~L}$ & 30 & $5 \mathrm{~N}$ & 30 & 5 & 15 & $100 \mathrm{~N}$ & $5 \mathrm{~L}$ & 50 & $100 \mathrm{~N}$ & $10 \mathrm{~L}$ & $50 \mathrm{~N}$ & 100 & $200 \mathrm{~N}$ & 150 & $.05 \mathrm{~N}$ \\
\hline 30 & 700 & 30 & $5 \mathrm{~N}$ & $20 \mathrm{~N}$ & 50 & $10 \mathrm{~L}$ & $100 \mathrm{~N}$ & 7 & 10 & 200 & 30 & $50 \mathrm{~N}$ & 30 & $200 \mathrm{~N}$ & 30 & $.05 \mathrm{~N}$ \\
\hline 70 & 30 & 50 & $5 \mathrm{~N}$ & $20 \mathrm{~L}$ & 20 & $10 \mathrm{~L}$ & $100 \mathrm{~N}$ & 7 & 150 & $100 \mathrm{~L}$ & 50 & $50 \mathrm{~L}$ & 15 & 300 & 100 & $.05 \mathrm{~N}$ \\
\hline 200 & 100 & 50 & $5 \mathrm{~N}$ & 20 & 70 & $10 \mathrm{~L}$ & $100 \mathrm{~N}$ & 20 & 100 & 700 & 200 & $50 \mathrm{~N}$ & 50 & 200 & 200 & $.05 \mathrm{~N}$ \\
\hline
\end{tabular}

Occurrences in and Adjacent to the Granite

Measurements were made on three samples from occurrences in and adjacent to the granite. Sample $33 \mathrm{~B}$ is a porphyritic felsic dike with phenocrysts of potassium feldspar, rounded quartz, and altered plagioclase set in a chloritic groundmass. The quartz phenocrysts contain liquid-rich fluid inclusions. Sample 032C is a sample of yellow-stained, porphyritic felsic dike that contains mostly small liquid-rich fluid inclusions. One or two vapor-rich inclusions were observed, and in one case a liquid-rich fluid-inclusion contains a daughter mineral (fig. $3 \mathrm{E}$ ). Sample 82380-21 is a sample of a felsic dike. Salinities for these samples range from 0.2 - to 8.0 -weight-percent $\mathrm{NaCl}$ equivalent, but most are lower than 2 percent. Heating data are shown as histograms (fig. 6). The histograms for the three samples are bimodal. The lower mode in two of the samples occurs at about 275 ${ }^{\circ} \mathrm{C}$; the lower mode in the third sample occurs at about $265^{\circ} \mathrm{C}$. The upper modes occur at 355,315 and 295
${ }^{\circ} \mathrm{C}$ in the three samples studied using the heatingfreezing stage. The data indicate that fluids were trapped at two temperatures, the lower of which was similar to that of type I inclusions in the quartz vein.

\section{Crushing Experiments}

A crushing stage was used to investigate the noncondensible gases in the fluid inclusions (Roedder, 1970 ; 1984). In a first test, materials from the six samples examined in the heating and cooling experiments were crushed in an immersion oil ( $\mathrm{D}=1.515$ ) mounting fluid. Upon crushing, gases were liberated from the fluid inclusions and formed bubbles in the oil. The gas bubbles moved into the liquid at moderate speeds. This test indicates that the vapor phase of the inclusions contains a noncondensible gas in addition to water vapor, and that the pressures inside the fluid inclusions are considerably above 1 atmosphere but are not so high as to cause the gases to 
Table 2. Analytical data of rocks from stations 31,32 , and 33 in the Table Mountain area collected in 1983

[Analysis of Au by atomic absorption; all other analyses by six-step semiquantitative emission spectrographic methods. $\mathrm{Fe}, \mathrm{Mg}, \mathrm{Ca}$, and $\mathrm{Ti}$ in percent; all others in parts per million. G, greater than the amount listed; $\mathrm{L}$, less than amount listed; N, not detected at amount listed. Analyst R.M. O'Leary ]

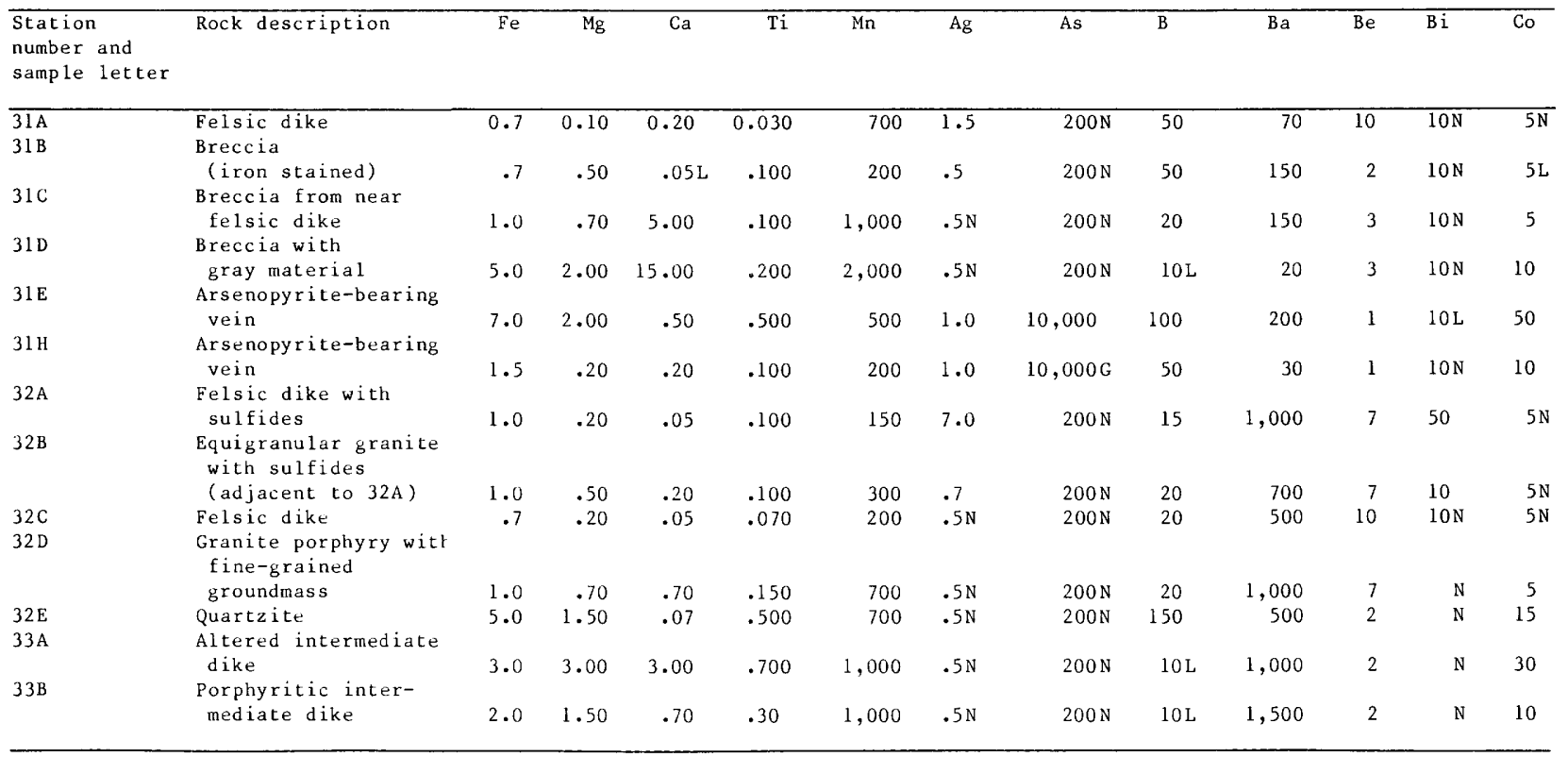

be expelled explosively. In order to determine if carbon dioxide is in the vapor phase of the fluid inclusions, a 10 percent barium chloride solution was used as a mounting fluid. Upon crushing, some of the vapor bubbles that were expelled from the fluid inclusions dissolved immediately into the barium chloride solution. When the mounting fluid was acidified by the addition of dilute hydrochloric acid, the bubbles that were expelled from the fluid inclusions did not disappear. The results indicate that carbon dioxide is present but distributed unevenly in the vapor phase of the fluid inclusions (see Roedder, 1984 , p. 212-219). However, carbon dioxide probably is not present in sufficient amounts to form a separate liquid carbon dioxide phase. The presence of the carbon dioxide in the fluids associated with mineralization calls into question depth estimates using a boiling $\mathrm{NaCl}-\mathrm{H}_{2} 0$ fluid (see Roedder, 1984). Nevertheless, based on geologic relations, the approximate depths calculated by the methods described by Haas (1971) are probably a good estimate for mineralization in the Table Mountain area.

\section{DISCUSSION}

The available data on the field relations, traceelement geochemistry, mineralogy, and fluid inclusions suggest (1) these occurrences, like other low-sulfide gold deposits and occurrences (see Boyle, 1979, p. 279290), have simple mineralogies that are associated with low-saline fluids having sulfur fugacities within the stability field of pyrrhotite; (2) the occurrences formed at moderate temperatures, and the fluids boiled; (3) the occurrences formed at relatively shallow depths; (4) the occurrences formed in an active tectonic environment; and (5) occurrences similar to these may have been the sources of placer gold in the Circle quadrangle.

The mineralogy and geochemistry of the occurrences near Table Mountain, as well as in and adjacent to the granite, are similar to other lowsulfide quartz vein deposits. The most common sulfides are arsenopyrite and pyrrhotite; minor sulfides and sulfosalts include enargite, chalcopyrite and sphalerite. Gold is associated with arsenic and is more abundant than silver. Studies of fluid inclusions suggest the fluids that deposited the metals were low salinity (10-weight-percent $\mathrm{NaCl}$ equivalent); the presence of pyrrhotite instead of pyrite constrains the sulfur fugacity of the fluid.

The occurrences formed at moderate temperatures and in part from fluids that boiled. Boiling is indicated by the presence of cogenetic liquid-rich and vapor-rich fluid inclusions in the quartz vein west of Table Mountain (station 31, fig. 4). The majority of filling temperatures of vapor-rich inclusions from this occurrence are from 320 to 380

${ }^{\circ} \mathrm{C}$. The average filling temperatures of liquid-rich fluid inclusions from this occurrence are from 255 to $275{ }^{\circ} \mathrm{C}$. Together, the fluid inclusions appear to record a cooling of fluids in the vein that was accompanied by the deposition of sulfides and quartz. Samples from occurrences in and adjacent to the granite (station 32,33 , and $82380-2$ ) do not contain vapor-rich fluid inclusions. Filling temperatures of liquid-rich fluid inclusions from samples of felsic dikes are bimodal. The upper temperature mode varies with the sample. The lower mode in the samples ranges from 265 to $275^{\circ} \mathrm{C}$, temperatures similar to those for samples of the quartz vein from near Table Mountain. 


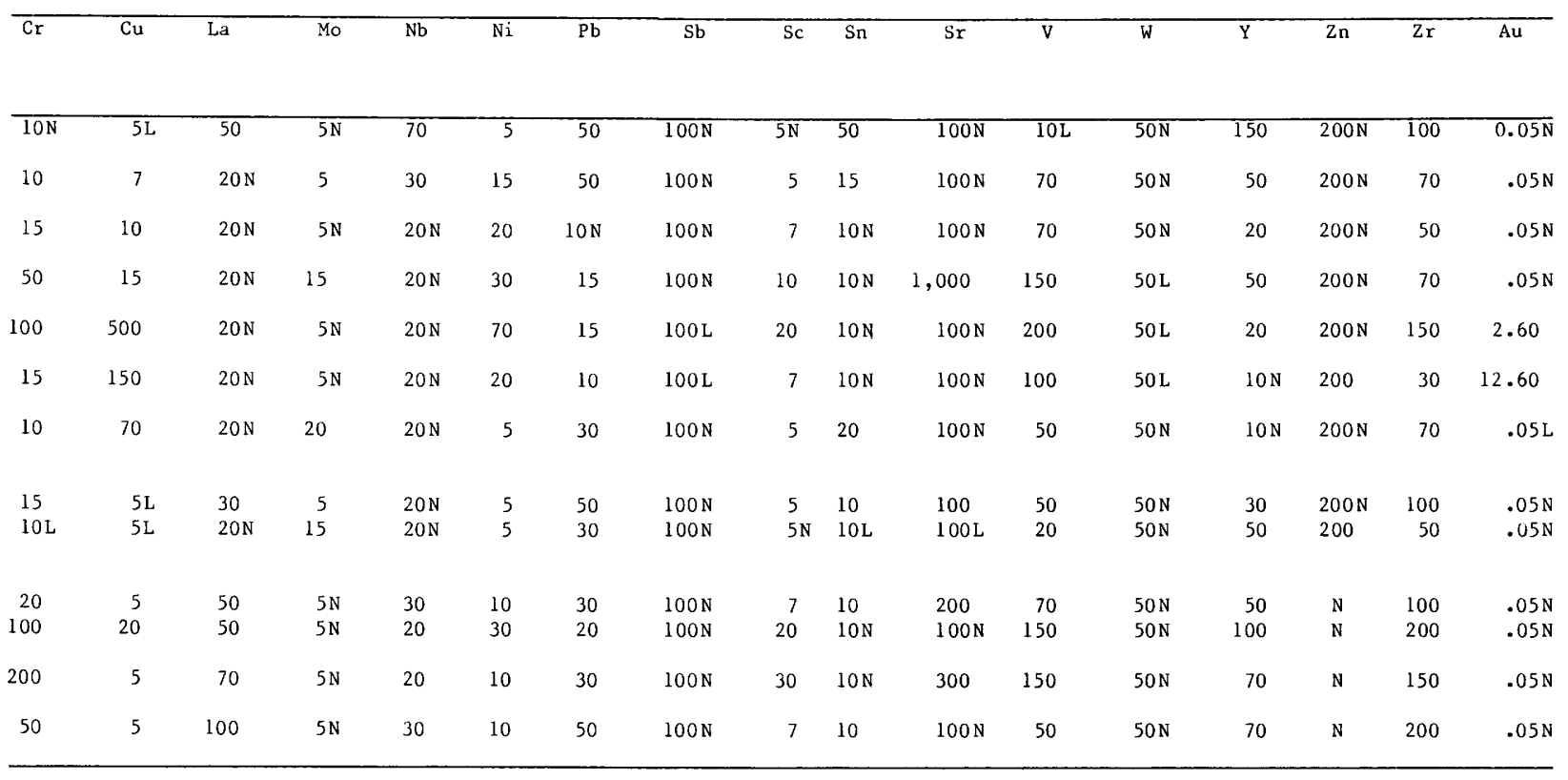

The inference that the occurrence formed at shallow depth is supported by two pieces of evidence: (1) using the method of Haas (1971), a fluid with a salinity of 5 weight percent and a temperature of 320 to $380^{\circ} \mathrm{C}$ will boil at a depth of 1,400 to $2,400 \mathrm{~m}$ if it is open to the surface. (2) The presence of detectable gold in felsic dikes (station 32,3103 and 31) and the presence of arsenopyrite in the granite adjacent to a felsic dike (station 32) suggests involvement of the dikes in the hydrothermal activity. The textures of the dikes suggest that they crystallized in a shallow environment.

The inference that the occurrences formed in the active tectonic environment that accompanied the late stages of the felsic igneous activity is supported by field relations, including probable movement on the Swamp Saddle fault. Differences in postulated depth of formation of the contact metamorphic effects and of the quartz vein west of Table Mountain provide additional evidence of tectonic activity.

Gold occurrences west of Table Mountain (stations 3095,3122 and 31 ) suggest control by the adjacent fault. The presence of tourmaline in the fault zone and in gold-bearing samples adjacent to the fault (stations 3095 and 3122), as well as locally from the outer walls of the quartz vein (station 31), links the fault and the gold-bearing samples.

The difference between the estimated depth of formation of the contact effects related to the intrusion of the granite $(6 \mathrm{~km})$, and the depth of formation of the quartz vein west of the Table Mountain as estimated from the method used by Haas (1971) (1.4 - $2.4 \mathrm{~km})$, implies substantial regional uplift. The time period over which the uplift took place is uncertain, because neither the granite nor the felsic dikes that are associated with the gold occurrences have been dated successfully. However, similar plutons in the northwest Circle quadrangle have yielded ages between 66 and $57 \mathrm{~m} . \mathrm{y}$. B.P. (Wilson and Shew, 1981). Felsic dikes and hypabyssal rocks similar to those in the Table Mountain area exist in or adjacent to other plutons in the region. The only occurrence of similar rocks that are not closely associated with a pluton occur in the Faith Creek area, where they occur along the trend of an aeromagnetic low that also underlies the Table Mountain area. These relationships suggest that the felsic dikes and hypabyssal rocks are comagmatic with the granite and place some constraints upon the time period over which the uplift took place. Thus it seems reasonable to suggest that the Table Mountain area was being uplifted at a rate of 3.6 to $4.6 \mathrm{~km}$ per $10 \mathrm{~m} . \mathrm{y}$. just before the start of the hydrothermal activity that formed the gold occurrences. This rate of uplift can be compared with the rate of $6 \mathrm{~km}$ in $60 \mathrm{~m} . \mathrm{y}$. that Forbes and Turner (1975) calculated for the Yukon Tanana Upland on the basis of argon-blocking temperatures in biotite.

The Swamp Saddle fault, which of fsets regional metamorphic isograds about $8 \mathrm{~km}$ (Foster and others, 1983) and of fsets dike rocks from the pluton, probably was a major locus of tectonic activity in the Table Mountain area.

The possibility that occurrences such as those west of Table Mountain (stations 3095, 3122 and 31) might act as sources of gold elsewhere in the Circle quadrangle is probably best assessed by estimating the amount of gold in the occurrences. If the quartz vein at station 31 does underlie the small hill on the west end of the ridge (an area of approximately $0.1 \mathrm{~km}^{2}$ ), averages $3.75 \mathrm{~cm}$ in thickness, and has an average gold content of $7 \mathrm{ppm}$, it would contain over 1,400 oz of 
$\boldsymbol{A}$

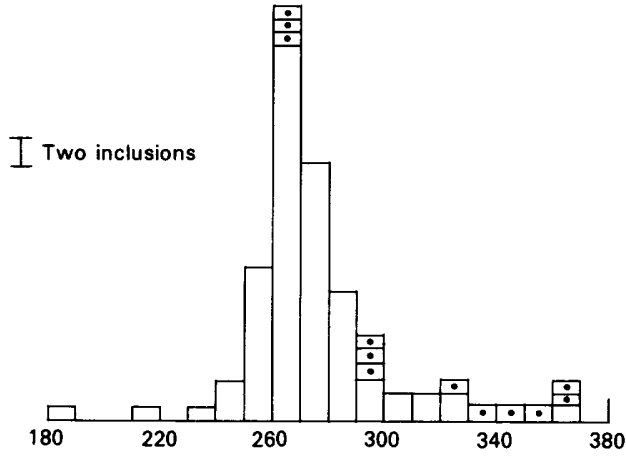

B
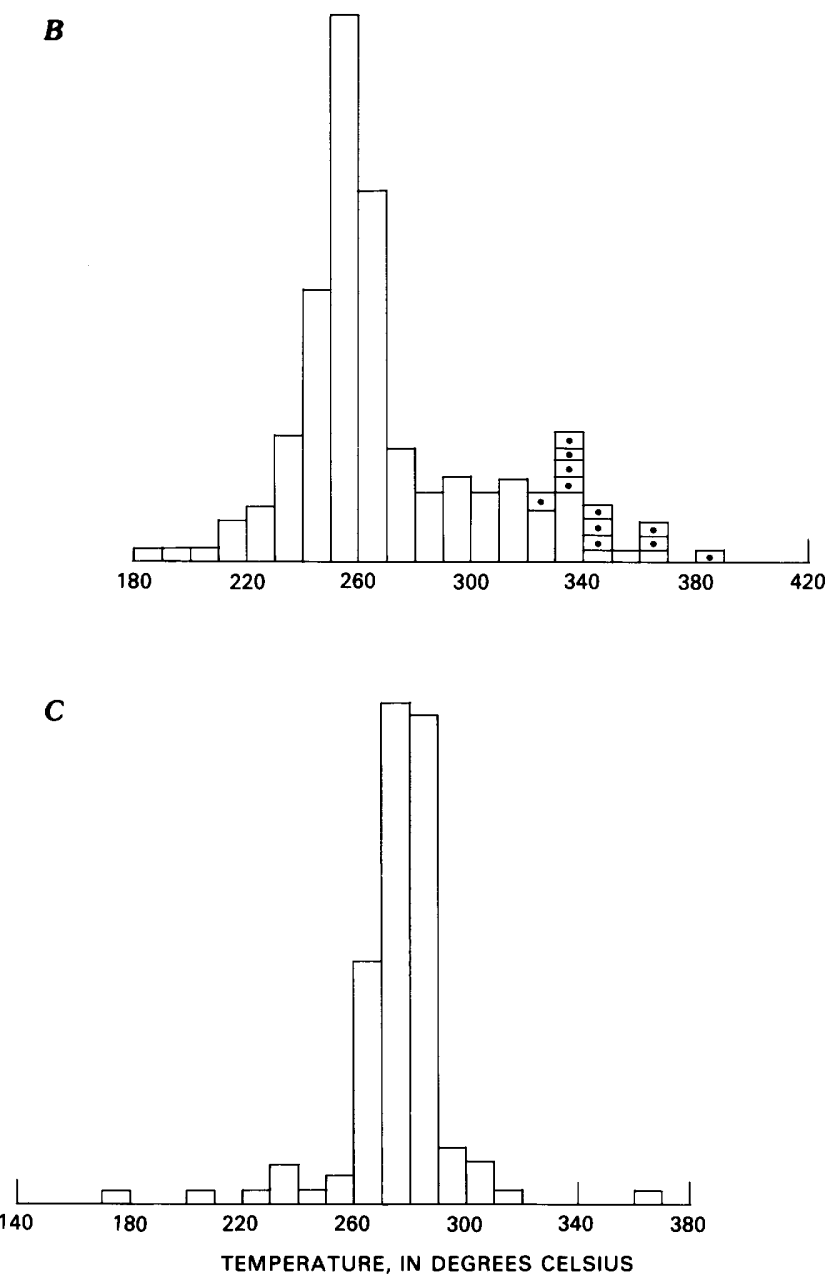

Figure 5. Histograms of homogenization temperatures of fluid inclusions from samples from Table Mountain occurrence. Open area, data for type I inclusion; area with dot, type II inclusion. A, Sample 31I. B, Sample

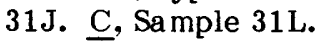
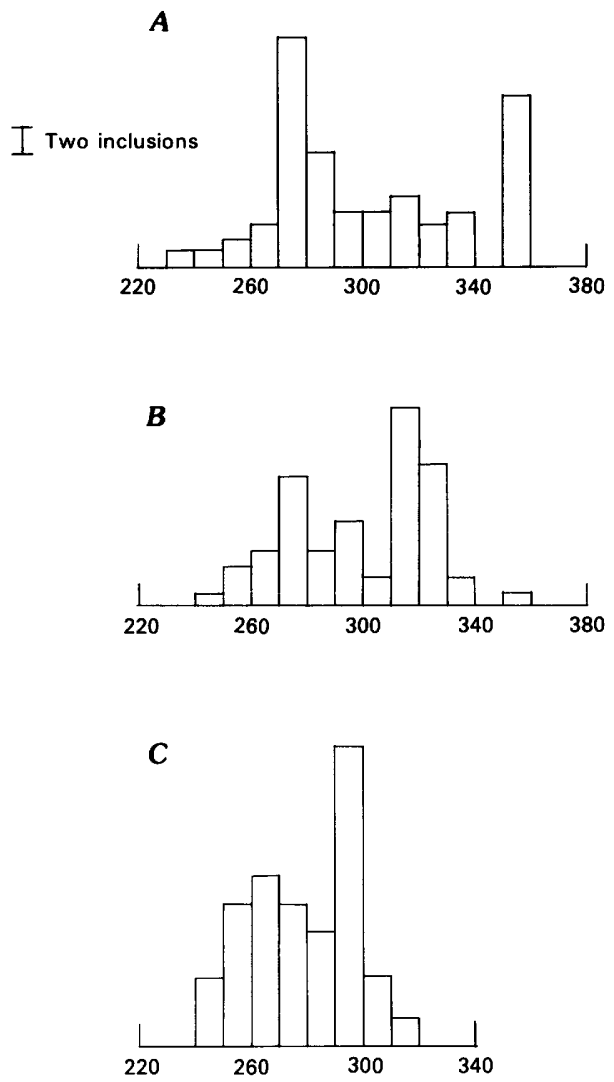

TEMPERATURE, IN DEGREES CELSIUS

Figure 6. Histograms of homogenization temperatures of fluid inclusions from samples from occurrences in and adjacent to the granite. All data are for type I inclusions. A, Sample 32C. B. Sample 33B. C., Sample 82380-2-1.

gold. Such a figure is probably a conservative estimate of the gold available in the area west of Table Mountain, because it neglects gold from the black biotite schist adjacent to the fault and does not consider the possibility of additional veins beneath the hill. Although the occurrence does not contain a large amount of gold, especially when compared to that mined in the Circle quadrangle, it seems likely that there were many similar occurrences within the quadrangle. Thus, much of the gold in the Circle quadrangle probably was derived from small local gold occurrences that are characterized by a simple mineralogy, and formed at shallow depths in and adjacent to faults that were active during the last stages of felsic igneous activity. 


\section{REFERENCES CITED}

Bodnar, R.J., Burnham, C.W., and Sterner, S.M., 1985, Synthetic fluid inclusions in natural quartz. III. Determination of phase equilibrium properities in the system $\mathrm{H}_{2} \mathrm{O}-\mathrm{NaCl}$ to $1000{ }^{\circ} \mathrm{C}$ and 1500 bars: Geochimica et Cosmochimica Acta, v. 49, p. 1861-1873.

Boyle, R.W., 1979, The geochemistry of gold and its deposits: Geological Survey of Canada Bulletin $280,584 \mathrm{p}$.

Bundtzen, T.K., Eakins, G.R., Clough, J.G., Lueck, L.L., Green, C.B., Robinson, M.S., and Coleman, D.A., 1984, Alaska's Mineral Industry 1983: Special Report 33; Alaska Division of Geological and Geophysical Surveys, $56 \mathrm{p}$.

Burack, A.C., 1983, Geology along the Pinnel Mountain trail, Circle quadrangle, Alaska; University of New Hampshire, Masters of Science thesis, $98 \mathrm{p}$.

Burack, A.C., Laird, Jo, Foster, H.L., and Cushing, G.W., 1984, Metamorphic petrology of the Table Mountain area, Circle quadrangle, Alaska, in Coonrad, W.L., and Elliott, R.L. eds., The United States Geological Survey in Alaska: Accomplishments during 1981: U.S. Geological Survey Circular 868, p. 54-57.

Forbes, R.B. and Turner, D.L., 1975, K-Ar studies in two deep basement drill-holes- A new estimate of argon blocking temperature for biotite: Annual Report of the Geophysical Institute, University of Alaska, p. 138-9.

Foster, H.L., Laird, Jo, Keith, T.E.L., Cushing, G.W., and Menzie, W.D., 1983, Preliminary geologic map of the Circle quadrangle, Alaska: U.S. Geological
Survey Open-File Report 83-170-A, 30 p.

Foster, H.L., O'Leary, R.M., McDougal, C.M., and Menzie, W.D., 1984, Analyses of rock samples from the Circle quadrangle, Alaska: U.S. Geological Survey Open-File Report 84-479, 120 p.

Haas, J.L., Jr., 1971, The effect of salinity on the maximum thermal gradient of a hydrothermal system at hydrostatic pressure: Economic Geology, v. 66, p. 940-946.

Menzie, W. D., Foster, H.L. , Tripp, R.B., and Yeend, W.E., 1983, Mineral resource assessment of the Circle quadrangle, Alaska: U.S. Geological Survey Open-File Report 83-170-B, 57 p.

Roedder, Edwin, 1962, Studies of fluid inclusions; I-low temperature application of a dual-purpose freezing and heating stage: Economic Geology, v. 57, p. 1045-1061.

1970, Applications of an improved crushing microscope stage to studies of the gases in fluid inclusions: Schweizerische, Mineralogische und Petrographische Mitteilungen, 50:00 Pt. 1, pp 4158.

1984, Fluid inclusions: Reviews in mineralogy; Mineralogical Society of America, v. 12, p. 212219.

Streckeisen, A., 1975, To each plutonic rock its proper name: Earth-Science Reviews, v. 12, p. 1-33.

Wilson, F.H., and Shew, Nora, 1981, Map and tables showing preliminary results of potassium-argon age studies in the Circle quadrangle, Alaska, with a compilation of previous dating work: U.S. Geological Survey Open-File Report 81-889, scale $1: 250,000,1$ sheet. 
